\title{
Measurement Properties of Existing Patient-Reported Outcome Measures on Medication Adherence: Systematic Review
}

Yu Heng Kwan ${ }^{1,2^{*}}$, PhD; Si Dun Weng ${ }^{2 *}$, BSc; Dionne Hui Fang Loh ${ }^{3 *}$, BEng; Jie Kie Phang ${ }^{4}$, BSc; Livia Jia Yi Oo ${ }^{2}$, BSc; Dan V Blalock ${ }^{5,6}$, PhD; Eng Hui Chew ${ }^{2}$, PhD; Kai Zhen Yap ${ }^{2}$, PhD; Corrinne Yong Koon Tan ${ }^{7}$, MBA; Sungwon Yoon $^{1,3}$, PhD; Warren Fong ${ }^{4,8,9}$, FAMS; Truls Østbye ${ }^{1 *}$, PhD; Lian Leng Low ${ }^{3,10,11^{*}}$, FCFP(S); Hayden Barry Bosworth $^{5,6,12,13^{*}}, \mathrm{PhD} ;$ Julian Thumboo ${ }^{1,4,9^{*}}$, FRCP

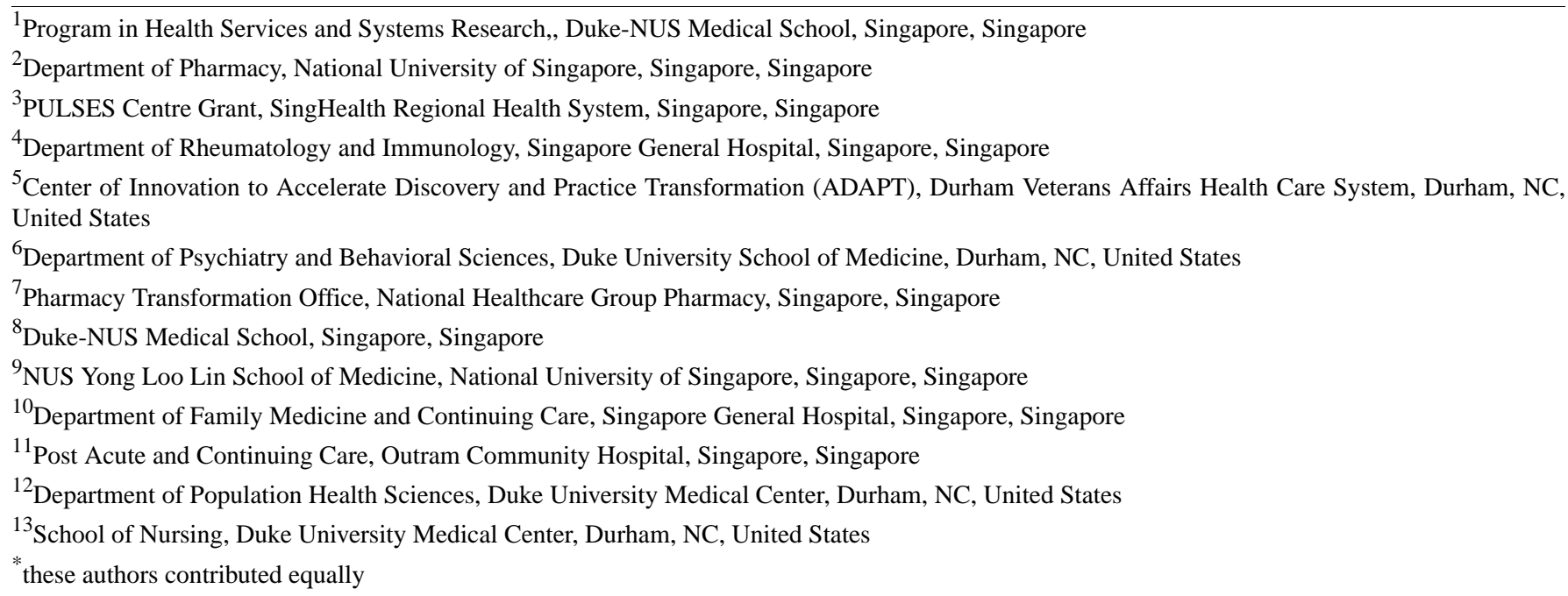

Corresponding Author:

Yu Heng Kwan, $\mathrm{PhD}$

Program in Health Services and Systems Research,

Duke-NUS Medical School

8 College Road

Singapore, 169857

Singapore

Phone: 6563778465

Email: yuheng_02071988@hotmail.com

\section{Abstract}

Background: Medication adherence is essential for improving the health outcomes of patients. Various patient-reported outcome measures (PROMs) have been developed to measure medication adherence in patients. However, no study has summarized the psychometric properties of these PROMs to guide selection for use in clinical practice or research.

Objective: This study aims to evaluate the quality of the PROMs used to measure medication adherence.

Methods: This study was guided by the PRISMA (Preferred Reporting Items for Systematic Review and Meta-Analysis) guidelines. Relevant articles were retrieved from the EMBASE, PubMed, Cochrane Library, Web of Science, and CINAHL (Cumulative Index to Nursing and Allied Health Literature) databases. The PROMs were then evaluated based on the COnsensus-based Standards for the selection of health Measurement Instruments (COSMIN) guidelines.

Results: A total of 121 unique medication adherence PROMs from 214 studies were identified. Hypotheses testing for construct validity and internal consistency were the most frequently assessed measurement properties. PROMs with at least a moderate level of evidence for $\geq 5$ measurement properties include the Adherence Starts with Knowledge 20, Compliance Questionnaire-Rheumatology, General Medication Adherence Scale, Hill-Bone Scale, Immunosuppressant Therapy Barrier Scale, Medication Adherence Reasons Scale (MAR-Scale) revised, 5-item Medication Adherence Rating Scale (MARS-5), 9-item MARS (MARS-9), 4-item Morisky Medication Adherence Scale (MMAS-4), 8-item MMAS (MMAS-8), Self-efficacy for 
Appropriate Medication Adherence Scale, Satisfaction with Iron Chelation Therapy, Test of Adherence to Inhalers, and questionnaire by Voils. The MAR-Scale revised, MMAS-4, and MMAS-8 have been administered electronically.

Conclusions: This study identified 121 PROMs for medication adherence and provided synthesized evidence for the measurement properties of these PROMs. The findings from this study may assist clinicians and researchers in selecting suitable PROMs to assess medication adherence.

(J Med Internet Res 2020;22(10):e19179) doi: $\underline{10.2196 / 19179}$

\section{KEYWORDS}

systematic review; reliability and validity; medication adherence; patient reported outcome measures

\section{Introduction}

Medication adherence is known as "the degree to which the person's behaviour corresponds with the agreed recommendations from a healthcare provider" [1]. Medication adherence is essential when it comes to improving the health outcomes of patients, especially for chronic diseases [2,3]. However, only approximately half of the patients worldwide adhere to their prescribed medication regimen [4]. Reasons for medication nonadherence include complexity of the treatment regimen, poor communication with health care providers, and concerns about side effects from taking medications [5]. Poor medication adherence may lead to worse health outcomes and higher rates of mortality and morbidity $[1,6]$. Nonadherence also incurs a high cost burden to the health care system by increasing hospital visits as well as causing unnecessary escalation to more expensive treatments [1]. Therefore, improving medication adherence is key to improving treatment outcomes $[7,8]$.

To successfully improve medication adherence, there is a need for the accurate assessment of medication adherence. Current practices for measuring medication include direct measures such as drug assays of blood or urine as well as indirect measures of adherence such as pill count, electronic monitoring devices, and the use of big data such as review of prescription records and claims $[9,10]$. Some of these measures are not time efficient and are likely to be unsustainable in clinical practice. Various patient-reported outcome measures (PROMs) such as the Morisky Medication Adherence Scale (MMAS) [11], Hill-Bone Compliance Scale [12], and Medication Adherence Rating Scale (MARS) [13] have been developed to measure self-reported adherence to medications. These PROMs may be useful in clinical practice because they are easy to administer. On the basis of the patients' PROM ratings, health care professionals may be able to provide timely feedback. Thus, underlying issues that contribute to medication noncompliance can be addressed at the point of care [14].

A number of previous studies have been conducted to validate PROMs on medication adherence [12,15-17], and a previous systematic review found 14 PROMs that assessed adherence to inhaled asthma maintenance medication alone [18]. However, to date, there is no comprehensive review that summarizes the psychometric properties of PROMs for medication adherence, which is essential to guide the selection of suitable PROMs to evaluate medication adherence in patients. Hence, we carried out a systematic review to identify studies that investigated
PROMs for medication adherence and to evaluate the quality of these PROMs.

\section{Methods}

This study was conducted with reference to the PRISMA (Preferred Reporting Items for Systematic Review and Meta-Analysis) statement [19]. The measurement properties of each PROM were evaluated based on the COnsensus-based Standards for the selection of health Measurement Instruments(COSMIN) guidelines [20,21]. The COSMIN guidelines evaluate PROM development and the following 9 measurement properties: "content validity," "structural validity," "internal consistency," "cross-cultural validity/measurement invariance," test-retest "reliability," "measurement error," "criterion validity," "hypotheses testing for construct validity," and "responsiveness" [21,22]. Of note, the assessment of "convergent validity," "discriminant validity," and "known-group validity" falls under "hypotheses testing for construct validity" $[23,24]$. We also assumed that "concurrent validity" and "predictive validity" can be evaluated by the same measurement property, "hypothesis testing for construct validity," and sensitivity to change can be evaluated under "responsiveness" as well [22,25,26].

\section{Search Strategy}

The EMBASE, PubMed, Cochrane Library, Web of Science, and CINAHL databases were searched for relevant studies published before November 1, 2019. A search strategy (Multimedia Appendix 1) consisting of adherence, PROMs, and measurement properties was used. Search filters created by Terwee et al [27], which consists of a combination of search terms, were also used to enhance the sensitivity of searches, where available. For the adherence construct, synonyms such as compliance were used in the search. Duplicates were removed from the final search library.

\section{Study Selection}

Articles included in this study were full-text publications in English, validating medication adherence PROMs, with the assessment of at least one measurement property listed in the COSMIN [24,28]. We excluded animal studies, case studies, narrative reviews, expert opinions, conference abstracts, and PROMs completed by proxy [29]. In total, 2 independent reviewers (SW and LO) screened the titles and abstracts of the studies according to the inclusion and exclusion criteria. Opinions from a third reviewer (YK) were sought in the event of any disagreements. The remaining full-text articles were then 
evaluated by the same 2 independent reviewers for inclusion and exclusion. Additional articles for inclusion into the final pool of articles were also identified from the reference list of articles during the full-text screening phase [30].

\section{Extraction of Data}

The following data were then extracted from the included articles by 2 independent reviewers ( $\mathrm{SW}$ and LO), where available:

- General characteristics of the study population: age, sample size, gender, and country.

- Characteristics of disease or condition: disease studied, duration of illness, or treatment.

- PROM characteristics: methods of administration, availability of electronic administration, language, response scale, domains, and number of items.

\section{Assessment of Methodological Quality}

The methodological quality of the studies was assessed by 2 independent reviewers (SW and LO). Any disagreement was resolved in consultation with a third reviewer (JP). Each measurement property was assessed based on a 4-point scale: "inadequate," "doubtful," "adequate," or "very good" [20,22]. The item with the worst rating under each measurement property would determine the overall rating for the specific measurement property [31].

On the basis of the COSMIN guidelines, it is recommended for the review team to determine before assessing the methodological quality of studies which outcome measurement instrument can be considered a reasonable gold standard [32]. The study team decided that there is currently no gold standard in the field of patient-reported outcomes that measure medication adherence $[33,34]$; thus, the assessment of criterion validity of the PROMs was not performed except when an abridged PROM was compared with the original long version, which will be regarded as the gold standard. This is in line with the consensus from the COSMIN panel that no gold standard exists for PROMs [35].

\section{Assessment of Psychometric Quality}

The psychometric quality for each medication adherence instrument was assessed using the quality criteria by Terwee et al [36]. For each of the measurement properties evaluated in the included studies, a "positive (+)," "indeterminate (?)," or "negative (-)" rating was assigned based on the psychometric results.For example, for the internal consistency measurement property, the rating will be "+" if "at least low evidence for sufficient structural validity AND Cronbach's alpha(s) $\geq 0.70$ for each unidimensional scale or subscale." The rating will be "_" if there is "at least low evidence for sufficient structural validity AND Cronbach's alpha(s) $<0.70$ for each unidimensional scale or subscale." The rating will be "?" if the "criteria for at least low evidence for sufficient structural validity is not met" [36].

\section{Evidence Synthesis}

For each PROM, an evidence synthesis across studies was conducted. First, we determined whether each measurement property for a PROM had overall "sufficient (+)," "insufficient $(-)$," "inconsistent $( \pm)$," or "indeterminate (?)" evidence for each measurement property of the PROM. For PROMs that were assessed in more than one study, the overall rating of the level of evidence for the PROM would be sufficient (+), indeterminate (?), or insufficient $(-)$ if the individual studies were all consistently rated as positive $(+)$, indeterminate (?), or negative (-), respectively. If the results of individual studies were inconsistent, the overall rating of the level of evidence for the PROM would be inconsistent.

We also graded the quality of evidence for each measurement property of PROM as "high," "moderate," "low," or "very low" level of evidence based on the guidelines from the modified Grading of Recommendations Assessment, Development and Evaluation approach for systematic reviews of clinical trials [22,37].

\section{Results}

\section{Search Results and Study Characteristics}

In total, 51,426 articles were retrieved from the 5 databases. After removing 8286 duplicates and 42,836 articles during title and abstract screening, 304 articles remained for full-text review. A total of 98 articles were further eliminated during full-text article screening. An additional 8 relevant articles were identified through hand-searching of the reference lists from the included articles to obtain a final list of 214 articles (Figure 1). A total of 240 PROMs were evaluated across 214 studies, and 121 unique medication adherence PROMs in 32 languages from 48 countries were identified (Table 1). 
Figure 1. Preferred Reporting Items for Systematic Reviews and Meta-Analyses flow diagram for the systematic review. CINAHL: Cumulative Index to Nursing and Allied Health Literature; PROM: patient-reported outcome measure.

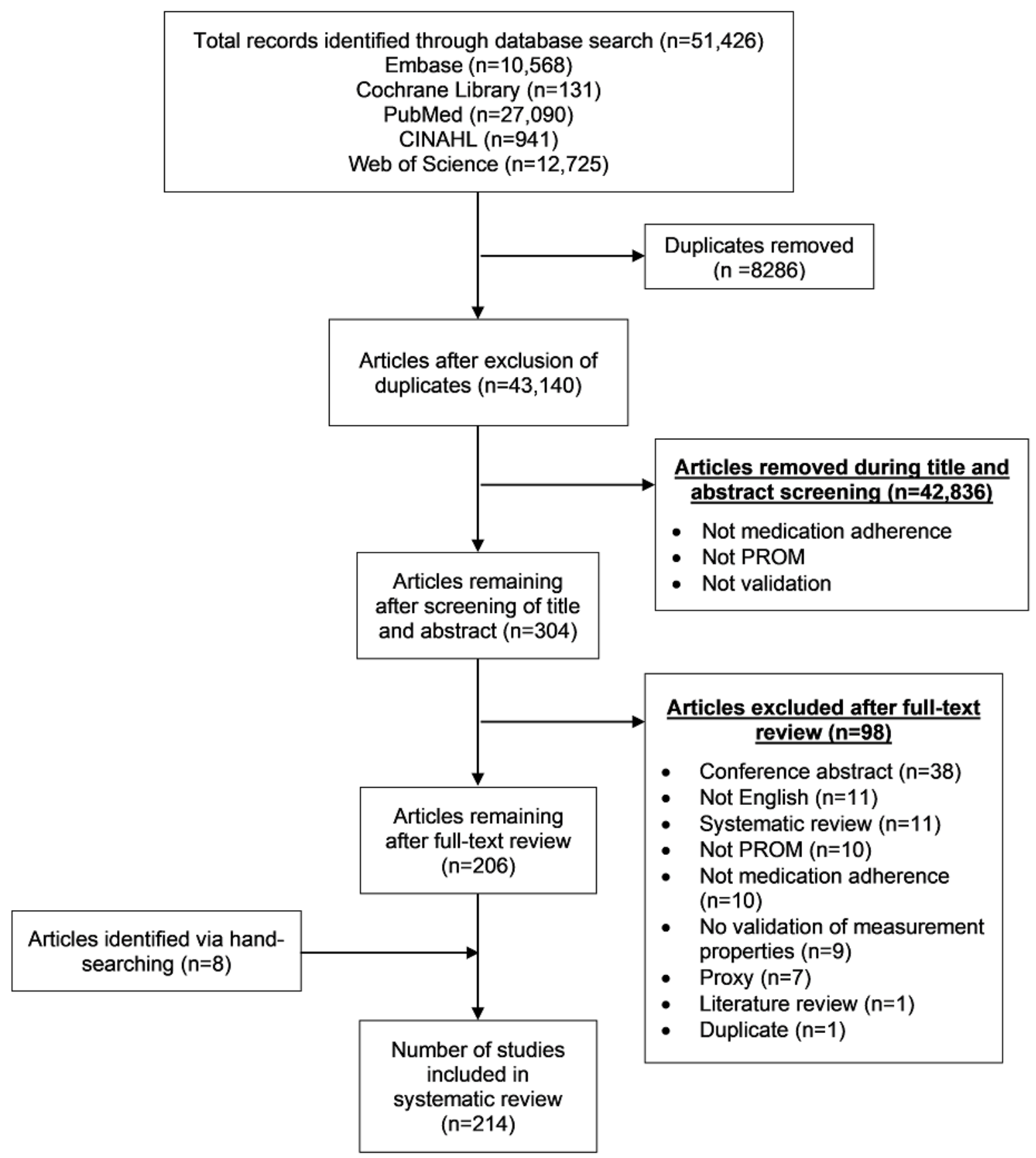


Table 1. Study characteristics of included articles $(\mathrm{N}=214)$.

\begin{tabular}{|c|c|}
\hline Study characteristics & Values, $\mathrm{n}(\%)$ \\
\hline \multicolumn{2}{|l|}{ Sample size $^{\mathrm{a}}$} \\
\hline$<50$ & $17(7.9)$ \\
\hline $50-99$ & $31(14.4)$ \\
\hline $100-199$ & $62(29.0)$ \\
\hline 200-299 & $32(15.0)$ \\
\hline $300-399$ & $23(10.7)$ \\
\hline $400-499$ & $15(7.0)$ \\
\hline$>500$ & $35(16.4)$ \\
\hline \multicolumn{2}{|l|}{ Mean age (years) ${ }^{\mathbf{a}, \mathbf{b}}$} \\
\hline$<20$ & $9(4.2)$ \\
\hline $20-39$ & $28(13.1)$ \\
\hline $40-59$ & $97(45.3)$ \\
\hline $60-79$ & $55(25.7)$ \\
\hline$>80$ & $2(0.9)$ \\
\hline \multicolumn{2}{|c|}{ Proportion of males $(\%)^{\mathrm{a}}$} \\
\hline $0-20$ & $15(7.0)$ \\
\hline $21-40$ & $47(22.0)$ \\
\hline $41-60$ & $78(36.4)$ \\
\hline $61-80$ & $38(17.8)$ \\
\hline $81-100$ & $23(10.7)$ \\
\hline \multicolumn{2}{|c|}{ Mean treatment/disease duration ${ }^{\mathbf{a}}$ (years) } \\
\hline$<1$ & $3(1.4)$ \\
\hline $1-5$ & $11(5.1)$ \\
\hline $6-10$ & 27 (12.6) \\
\hline $11-15$ & $16(7.5)$ \\
\hline$>15$ & $2(0.9)$ \\
\hline
\end{tabular}

${ }^{\mathrm{a}}$ Some values were not reported.

${ }^{\mathrm{b}}$ Some values were reported as median, range, or not reported.

\section{PROM Characteristics}

The characteristics of the medication adherence PROM are presented in Multimedia Appendix 2 [38-51]. The most extensively studied PROMs were the 8-item MMAS (MMAS-8)and 4-item MMAS(MMAS-4), which were studied in 27 and 18 studies, respectively. Among the 121 PROMs, 15 PROMs have been administered electronically-Adult AIDS Clinical Trials Group (AACTG) adherence items [52], Fredericksen et al [38], Item Response Theory-30 [53], LeastAbsolute Shrinkage and Selection Operator-10 [53], Medication Adherence Reasons Scale (MAR-Scale) [54], MAR-Scale (revised) [55], MARS [56], Medication Intake Survey-Asthma [57], MMAS-4 [54], MMAS-8 [58], Multiple Sclerosis Treatment Adherence Questionnaire [59], Probabilistic Medication Adherence Scale [56], Self-Rating Scale Item [52], visual analog scale [52], and WedAd-Q Questionnaire [60].

\section{Assessment of Methodological and Psychometric Quality}

Outcomes on the assessment of methodological quality and study quality of PROMs are summarized in Multimedia Appendix 3 [13,17,38-54,56,57,59,61-248]. In terms of validity, "hypotheses testing for construct validity,"“structural validity," and "content validity" were assessed in 181, 92, and 92 studies, respectively. In terms of reliability, "internal consistency" and "reliability" were assessed in 138 and 27 studies, respectively.

No studies assessed "measurement error" or "cross-cultural validity/measurement invariance.”Of note, 46 studies performed translation of PROMs, but none of them assessed measurement invariance or differential item functioning. Furthermore, although 75 studies assessed PROM development, only one of them obtained very good methodological quality. 


\section{Evidence Synthesis}

The results for the evidence synthesis for the PROMs are summarized in Table 2. PROMs with at least a moderate level of evidence for $\geq 5$ measurement properties include the Adherence Starts with Knowledge 20 (ASK-20), Compliance Questionnaire-Rheumatology (CQR), General Medication
Adherence Scale (GMAS), Hill-Bone Scale, Immunosuppressant Therapy Barrier Scale (ITBS), MAR-Scale revised, MARS-5, MARS-9, MMAS-4, MMAS-8, Self-efficacy for Appropriate Medication Adherence Scale (SEAMS), Satisfaction with Iron Chelation Therapy (SICT), Test of Adherence to Inhalers (TAI), and the questionnaire by Voils. 
Table 2. Evidence synthesis of measurement properties for each patient-reported outcome measure.

\begin{tabular}{|c|c|c|c|c|c|c|c|c|c|c|c|}
\hline $\mathrm{PROM}^{\mathrm{a}}$ & $\begin{array}{l}\text { Num- } \\
\text { ber of } \\
\text { studies }\end{array}$ & $\begin{array}{l}\text { PROM } \\
\text { develop- } \\
\text { ment }\end{array}$ & $\begin{array}{l}\text { Con- } \\
\text { tent } \\
\text { validi- } \\
\text { ty }\end{array}$ & $\begin{array}{l}\text { Struc- } \\
\text { tural va- } \\
\text { lidity }\end{array}$ & $\begin{array}{l}\text { Internal } \\
\text { consisten- } \\
\text { cy }\end{array}$ & $\begin{array}{l}\text { Cross-cultur- } \\
\text { al validi- } \\
\text { ty/measure- } \\
\text { ment invari- } \\
\text { ance }\end{array}$ & $\begin{array}{l}\text { Reliabil- } \\
\text { ity }\end{array}$ & $\begin{array}{l}\text { Mea- } \\
\text { sure- } \\
\text { ment er- } \\
\text { ror }\end{array}$ & $\begin{array}{l}\text { Criteri- } \\
\text { on valid- } \\
\text { ity }\end{array}$ & $\begin{array}{l}\text { Hypothe- } \\
\text { ses test- } \\
\text { ing for } \\
\text { construct } \\
\text { validity }\end{array}$ & $\begin{array}{l}\text { Responsive- } \\
\text { ness }\end{array}$ \\
\hline $\begin{array}{l}\text { Adult Asthma Adher- } \\
\text { ence Questionnaire }\end{array}$ & 1 & $0^{\mathrm{b}}$ & $?^{\mathrm{c}} / \mathrm{B}^{\mathrm{d}}$ & ?/B & $-{ }^{\mathrm{e}} / \mathrm{C}^{\mathrm{f}}$ & 0 & 0 & 0 & 0 & $-/ \mathrm{B}$ & $++^{g} / B$ \\
\hline $\begin{array}{l}\text { Adult AIDS Clinical } \\
\text { Trials Group }\end{array}$ & 8 & 0 & $? / \mathrm{D}^{\mathrm{h}}$ & ?/B & $+/ \mathrm{A}^{\mathrm{i}}$ & 0 & 0 & 0 & 0 & $\pm^{\mathrm{j}} / \mathrm{C}$ & $+/ \mathrm{C}$ \\
\hline $\begin{array}{l}\text { Antidepressant Adher- } \\
\text { ence Scale }\end{array}$ & 1 & D & $? / \mathrm{D}$ & 0 & $-/ \mathrm{D}$ & 0 & 0 & 0 & 0 & $-/ \mathrm{C}$ & 0 \\
\hline $\begin{array}{l}\text { Adherence Barrier } \\
\text { Questionnaire }\end{array}$ & 2 & $\mathrm{D}$ & 0 & ?/B & $+/ \mathrm{A}$ & 0 & 0 & 0 & 0 & $-/ \mathrm{B}$ & 0 \\
\hline $\begin{array}{l}\text { Adherence tool for } \\
\text { chronic myelomonocyt- } \\
\text { ic leukemia }\end{array}$ & 1 & D & 0 & 0 & $-/ \mathrm{C}$ & 0 & 0 & 0 & 0 & $-/ \mathrm{D}$ & $-/ \mathrm{D}$ \\
\hline $\begin{array}{l}\text { Adherence Evaluation } \\
\text { of Osteoporosis Treat- } \\
\text { ment Questionnaire-12 }\end{array}$ & 1 & 0 & 0 & 0 & 0 & 0 & 0 & 0 & 0 & $+/ \mathrm{A}$ & $+/ \mathrm{A}$ \\
\hline $\begin{array}{l}\text { Antipsychotic Medica- } \\
\text { tion Beliefs and Atti- } \\
\text { tudes Scale }\end{array}$ & 1 & $\mathrm{D}$ & $+/ \mathrm{C}$ & $-/ \mathrm{B}$ & $+/ \mathrm{A}$ & 0 & 0 & 0 & 0 & $+/ \mathrm{B}$ & 0 \\
\hline $\begin{array}{l}\text { Adherence to Pul- } \\
\text { monary Rehabilitation } \\
\text { Questionnaire }\end{array}$ & 1 & $\mathrm{C}$ & $+/ \mathrm{C}$ & ?/B & $+/ \mathrm{D}$ & 0 & 0 & 0 & 0 & 0 & 0 \\
\hline $\mathrm{ARMS}^{\mathrm{k}}$ & 1 & $\mathrm{C}$ & $+/ \mathrm{C}$ & ?/B & $+/ \mathrm{A}$ & 0 & ?/C & 0 & 0 & $+/ \mathrm{B}$ & 0 \\
\hline ARMS-7 & 1 & 0 & $? / \mathrm{C}$ & $-/ \mathrm{A}$ & $+/ \mathrm{A}$ & 0 & $+/ \mathrm{B}$ & 0 & 0 & 0 & 0 \\
\hline $\mathrm{ASK}^{1}-12$ & 1 & 0 & 0 & ?/B & $+/ \mathrm{A}$ & 0 & $+/ \mathrm{B}$ & 0 & 0 & $+/ \mathrm{A}$ & 0 \\
\hline ASK-20 & 3 & B & $+/ \mathrm{B}$ & ?/B & $+/ \mathrm{D}$ & 0 & $+/ \mathrm{B}$ & 0 & 0 & $\pm / \mathrm{A}$ & $-/ \mathrm{B}$ \\
\hline $\begin{array}{l}\text { Attitudes to mesalamine } \\
\text { questionnaire }\end{array}$ & 1 & D & 0 & 0 & 0 & 0 & 0 & 0 & 0 & $+/ \mathrm{A}$ & $+/ \mathrm{A}$ \\
\hline $\begin{array}{l}\text { Adherence self-report } \\
\text { questionnaire }\end{array}$ & 1 & 0 & $+/ \mathrm{C}$ & 0 & 0 & 0 & $-/ \mathrm{D}$ & 0 & 0 & 0 & $-/ \mathrm{B}$ \\
\hline 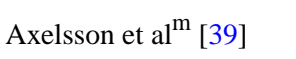 & 1 & D & 0 & ?/B & $+/ \mathrm{A}$ & 0 & 0 & 0 & 0 & $+/ \mathrm{C}$ & 0 \\
\hline $\begin{array}{l}\text { Basel Assessment of } \\
\text { Adherence to Immuno- } \\
\text { suppressive Medica- } \\
\text { tions Scale }\end{array}$ & 1 & 0 & $+/ \mathrm{B}$ & ?/B & $+/ \mathrm{D}$ & ${ }^{\mathrm{n}}$ & $+/ \mathrm{A}$ & 0 & 0 & $+/ \mathrm{B}$ & 0 \\
\hline $\begin{array}{l}\text { Brief Evaluation of } \\
\text { Medication Influences } \\
\text { and Beliefs }\end{array}$ & 1 & D & ?/D & $? / \mathrm{C}$ & $-/ \mathrm{D}$ & 0 & $? / \mathrm{D}$ & 0 & 0 & $-/ \mathrm{D}$ & $+/ \mathrm{C}$ \\
\hline $\begin{array}{l}\text { Beliefs Related to Med- } \\
\text { ication Adherence }\end{array}$ & 1 & D & ?/D & $? / \mathrm{D}$ & $+/ \mathrm{D}$ & 0 & 0 & 0 & 0 & $+/ \mathrm{D}$ & 0 \\
\hline $\begin{array}{l}\text { Brief Medication Adher- } \\
\text { ence Scale }\end{array}$ & 1 & D & 0 & ?/B & $? / \mathrm{D}$ & 0 & $+/ \mathrm{B}$ & 0 & 0 & $+/ \mathrm{C}$ & 0 \\
\hline $\begin{array}{l}\text { Beliefs about Medica- } \\
\text { tion Compliance Scale }\end{array}$ & 1 & $\mathrm{D}$ & $+/ \mathrm{C}$ & ?/B & $+/ \mathrm{A}$ & 0 & 0 & 0 & 0 & 0 & 0 \\
\hline $\begin{array}{l}\text { Brief Medication Ques- } \\
\text { tionnaire }\end{array}$ & 4 & D & ?/C & 0 & 0 & 0 & $+/ \mathrm{B}$ & 0 & 0 & $+/ \mathrm{B}$ & $+/ \mathrm{C}$ \\
\hline $\begin{array}{l}\text { Center for Adherence } \\
\text { Support Evaluation Ad- } \\
\text { herence Index }\end{array}$ & 2 & 0 & 0 & 0 & 0 & $0^{\mathrm{n}}$ & $? / \mathrm{D}$ & 0 & 0 & $+/ \mathrm{B}$ & $-/ \mathrm{B}$ \\
\hline
\end{tabular}




\begin{tabular}{|c|c|c|c|c|c|c|c|c|c|c|c|}
\hline $\mathrm{PROM}^{\mathrm{a}}$ & $\begin{array}{l}\text { Num- } \\
\text { ber of } \\
\text { studies }\end{array}$ & $\begin{array}{l}\text { PROM } \\
\text { develop- } \\
\text { ment }\end{array}$ & $\begin{array}{l}\text { Con- } \\
\text { tent } \\
\text { validi- } \\
\text { ty }\end{array}$ & $\begin{array}{l}\text { Struc- } \\
\text { tural va- } \\
\text { lidity }\end{array}$ & $\begin{array}{l}\text { Internal } \\
\text { consisten- } \\
\text { cy }\end{array}$ & $\begin{array}{l}\text { Cross-cultur- } \\
\text { al validi- } \\
\text { ty/measure- } \\
\text { ment invari- } \\
\text { ance }\end{array}$ & $\begin{array}{l}\text { Reliabil- } \\
\text { ity }\end{array}$ & $\begin{array}{l}\text { Mea- } \\
\text { sure- } \\
\text { ment er- } \\
\text { ror }\end{array}$ & $\begin{array}{l}\text { Criteri- } \\
\text { on valid- } \\
\text { ity }\end{array}$ & $\begin{array}{l}\text { Hypothe- } \\
\text { ses test- } \\
\text { ing for } \\
\text { construct } \\
\text { validity }\end{array}$ & $\begin{array}{l}\text { Responsive- } \\
\text { ness }\end{array}$ \\
\hline $\begin{array}{l}\text { Chronic Disease Com- } \\
\text { pliance Instrument }\end{array}$ & 3 & $\mathrm{~A}$ & $+/ \mathrm{A}$ & $? / \mathrm{B}$ & $+/ \mathrm{B}$ & $-^{n}$ & 0 & 0 & 0 & $-/ \mathrm{B}$ & 0 \\
\hline CEAT-VIH & 1 & 0 & $+/ \mathrm{B}$ & 0 & $+/ \mathrm{D}$ & 0 & 0 & 0 & 0 & $+/ \mathrm{C}$ & 0 \\
\hline Chaiyachatiet $\mathrm{al}^{\mathrm{m}}$ [40] & 1 & 0 & 0 & 0 & 0 & 0 & 0 & 0 & 0 & 0 & $-/ \mathrm{C}$ \\
\hline Compliance assessment & 1 & 0 & 0 & 0 & $? / \mathrm{D}$ & 0 & 0 & 0 & 0 & $+/ \mathrm{B}$ & 0 \\
\hline $\begin{array}{l}\text { Cohort Study of Medica- } \\
\text { tion Adherence Among } \\
\text { Older Adults self-report } \\
\text { tool }\end{array}$ & 1 & $\mathrm{D}$ & 0 & $? / \mathrm{D}$ & 0 & 0 & 0 & 0 & 0 & $+/ \mathrm{B}$ & $+/ \mathrm{B}$ \\
\hline $\mathrm{CQR}^{\mathrm{O}}$ & 4 & B & $+/ \mathrm{B}$ & $? / \mathrm{B}$ & $+/ \mathrm{A}$ & $0^{\mathrm{n}}$ & $\pm / \mathrm{B}$ & 0 & 0 & $\pm / \mathrm{B}$ & $+/ \mathrm{A}$ \\
\hline CQR-5 & 1 & 0 & $+/ \mathrm{A}$ & $+/ \mathrm{D}$ & $+/ \mathrm{A}$ & 0 & 0 & 0 & 0 & $+/ \mathrm{B}$ & $-/ \mathrm{B}$ \\
\hline Da et $\mathrm{al}^{\mathrm{m}}[41]$ & 1 & 0 & 0 & 0 & 0 & 0 & 0 & 0 & 0 & $-/ \mathrm{C}$ & $-/ \mathrm{B}$ \\
\hline $\mathrm{DAI}^{\mathrm{p}}$ & 1 & $\mathrm{D}$ & 0 & 0 & $+/ \mathrm{D}$ & 0 & $? / \mathrm{D}$ & 0 & 0 & $+/ \mathrm{B}$ & 0 \\
\hline DAI-10 & 2 & 0 & 0 & 0 & $+/ \mathrm{C}$ & 0 & 0 & 0 & 0 & $\pm / \mathrm{A}$ & $-/ \mathrm{B}$ \\
\hline DAI-9 & 1 & 0 & 0 & $? / \mathrm{C}$ & 0 & 0 & $-/ \mathrm{D}$ & 0 & 0 & $-/ \mathrm{D}$ & 0 \\
\hline $\begin{array}{l}\text { Diagnostic Adherence } \\
\text { to Medication Scale }\end{array}$ & 1 & B & $+/ \mathrm{B}$ & 0 & 0 & 0 & 0 & 0 & 0 & $+/ \mathrm{A}$ & 0 \\
\hline Demirtas et $\mathrm{al}^{\mathrm{m}}$ [42] & 1 & B & $+/ \mathrm{B}$ & ?/A & $+/ \mathrm{A}$ & 0 & $? / \mathrm{C}$ & 0 & 0 & $+/ \mathrm{C}$ & 0 \\
\hline $\begin{array}{l}\text { Danish version of Med- } \\
\text { ication Adherence Re- } \\
\text { port Scale- } 4\end{array}$ & 1 & 0 & $? / \mathrm{D}$ & 0 & $+/ \mathrm{C}$ & 0 & 0 & 0 & 0 & $-/ \mathrm{D}$ & 0 \\
\hline $\begin{array}{l}\text { Diabetes Management } \\
\text { Questionnaire }\end{array}$ & 1 & $\mathrm{D}$ & $? / \mathrm{C}$ & 0 & $+/ \mathrm{A}$ & 0 & $-/ \mathrm{C}$ & 0 & 0 & $+/ \mathrm{B}$ & 0 \\
\hline $\begin{array}{l}\text { Diabetes Medication } \\
\text { Self-efficacy Scale }\end{array}$ & 2 & $\mathrm{D}$ & $+/ \mathrm{B}$ & ?/B & $+/ \mathrm{D}$ & $0^{\mathrm{n}}$ & $+/ \mathrm{B}$ & 0 & 0 & $+/ \mathrm{C}$ & $-/ \mathrm{B}$ \\
\hline $\begin{array}{l}\text { Environmental Barriers } \\
\text { to Adherence Scale }\end{array}$ & 1 & 0 & 0 & 0 & $+/ \mathrm{A}$ & 0 & $? / \mathrm{D}$ & 0 & 0 & $+/ \mathrm{D}$ & 0 \\
\hline $\begin{array}{l}\text { Eye-Drop Satisfaction } \\
\text { Questionnaire }\end{array}$ & 2 & $\mathrm{D}$ & $+/ \mathrm{C}$ & ?/B & $+/ \mathrm{A}$ & 0 & 0 & 0 & 0 & $? / \mathrm{C}$ & 0 \\
\hline $\begin{array}{l}\text { End-Stage Renal Dis- } \\
\text { ease Adherence Ques- } \\
\text { tionnaire }\end{array}$ & 2 & $\mathrm{D}$ & $+/ \mathrm{B}$ & 0 & 0 & $0^{\mathrm{n}}$ & $+/ \mathrm{C}$ & 0 & 0 & $-/ \mathrm{B}$ & 0 \\
\hline $\begin{array}{l}\text { Every Visit Adherence } \\
\text { Questionnaire }\end{array}$ & 1 & 0 & 0 & 0 & 0 & 0 & 0 & 0 & 0 & $+/ \mathrm{D}$ & 0 \\
\hline $\begin{array}{l}\text { Five-dimension adher- } \\
\text { ence model }\end{array}$ & 1 & $\mathrm{D}$ & 0 & 0 & 0 & 0 & 0 & 0 & 0 & 0 & $+/ \mathrm{A}$ \\
\hline $\begin{array}{l}\text { Fredericksen et } \mathrm{al}^{\mathrm{m}} \\
\text { [249] }\end{array}$ & 1 & $\mathrm{C}$ & $+/ \mathrm{A}$ & 0 & 0 & 0 & $+/ \mathrm{A}$ & 0 & 0 & 0 & 0 \\
\hline $\begin{array}{l}\text { General adherence ten- } \\
\text { dency measure }\end{array}$ & 1 & $\mathrm{D}$ & 0 & 0 & 0 & 0 & 0 & 0 & 0 & $-/ \mathrm{B}$ & $-/ \mathrm{B}$ \\
\hline $\begin{array}{l}\text { General Medicine Ad- } \\
\text { herence Scale }\end{array}$ & 3 & $\mathrm{C}$ & $+/ \mathrm{B}$ & $+/ \mathrm{A}$ & $+/ \mathrm{A}$ & $0^{\mathrm{n}}$ & $\pm / \mathrm{B}$ & 0 & 0 & $+/ \mathrm{B}$ & $+/ \mathrm{C}$ \\
\hline Godin et $\mathrm{al}^{\mathrm{m}}$ [43] & 2 & $\mathrm{C}$ & $? / \mathrm{C}$ & 0 & 0 & 0 & 0 & 0 & 0 & $-/ \mathrm{C}$ & $+/ \mathrm{C}$ \\
\hline GTCAT $^{\mathrm{q}}$ & 1 & $\mathrm{D}$ & ?/B & $? / \mathrm{D}$ & $-/ \mathrm{B}$ & 0 & $? / \mathrm{D}$ & 0 & 0 & $+/ \mathrm{B}$ & 0 \\
\hline GTCAT (reduced) & 2 & 0 & ?/B & $-/ \mathrm{C}$ & $-/ \mathrm{A}$ & 0 & $+/ \mathrm{B}$ & 0 & 0 & $-/ \mathrm{B}$ & 0 \\
\hline
\end{tabular}




\begin{tabular}{|c|c|c|c|c|c|c|c|c|c|c|c|}
\hline $\mathrm{PROM}^{\mathrm{a}}$ & $\begin{array}{l}\text { Num- } \\
\text { ber of } \\
\text { studies }\end{array}$ & $\begin{array}{l}\text { PROM } \\
\text { develop- } \\
\text { ment }\end{array}$ & $\begin{array}{l}\text { Con- } \\
\text { tent } \\
\text { validi- } \\
\text { ty }\end{array}$ & $\begin{array}{l}\text { Struc- } \\
\text { tural va- } \\
\text { lidity }\end{array}$ & $\begin{array}{l}\text { Internal } \\
\text { consisten- } \\
\text { cy }\end{array}$ & $\begin{array}{l}\text { Cross-cultur- } \\
\text { al validi- } \\
\text { ty/measure- } \\
\text { ment invari- } \\
\text { ance }\end{array}$ & $\begin{array}{l}\text { Reliabil- } \\
\text { ity }\end{array}$ & $\begin{array}{l}\text { Mea- } \\
\text { sure- } \\
\text { ment er- } \\
\text { ror }\end{array}$ & $\begin{array}{l}\text { Criteri- } \\
\text { on valid- } \\
\text { ity }\end{array}$ & $\begin{array}{l}\text { Hypothe- } \\
\text { ses test- } \\
\text { ing for } \\
\text { construct } \\
\text { validity }\end{array}$ & $\begin{array}{l}\text { Responsive- } \\
\text { ness }\end{array}$ \\
\hline Hill-Bone Scale & 5 & B & $+/ \mathrm{A}$ & ?/B & $+/ \mathrm{B}$ & $0^{\mathrm{n}}$ & 0 & 0 & 0 & $+/ \mathrm{A}$ & 0 \\
\hline $\begin{array}{l}\text { Hill-Bone Scale (modi- } \\
\text { fied) }\end{array}$ & 1 & 0 & $? / \mathrm{C}$ & 0 & $-/ \mathrm{D}$ & $0^{\mathrm{n}}$ & 0 & 0 & 0 & 0 & 0 \\
\hline HIV Intention Measure & 1 & $\mathrm{C}$ & $+/ \mathrm{B}$ & ?/B & $+/ \mathrm{A}$ & 0 & 0 & 0 & 0 & $+/ \mathrm{C}$ & 0 \\
\hline $\begin{array}{l}\text { HIV Symptom Quality } \\
\text { of Life Adherence } \\
\text { Questionnaire }\end{array}$ & 1 & $\mathrm{D}$ & 0 & ?/B & $+/ \mathrm{C}$ & 0 & 0 & 0 & 0 & $+/ \mathrm{B}$ & 0 \\
\hline $\begin{array}{l}\text { Iraqi Anti-Diabetic } \\
\text { Medication Adherence } \\
\text { Scale }\end{array}$ & 1 & $\mathrm{C}$ & $? / \mathrm{C}$ & 0 & $+/ \mathrm{B}$ & $0^{\mathrm{n}}$ & $? / \mathrm{C}$ & 0 & 0 & $+/ \mathrm{B}$ & $+/ \mathrm{C}$ \\
\hline $\begin{array}{l}\text { Item Response Theory- } \\
30\end{array}$ & 1 & B & $+/ \mathrm{B}$ & $? / \mathrm{B}$ & 0 & 0 & 0 & 0 & 0 & $? / \mathrm{C}$ & $+/ \mathrm{B}$ \\
\hline $\begin{array}{l}\text { Immunosuppressant } \\
\text { Therapy Adherence } \\
\text { Scale }\end{array}$ & 2 & 0 & 0 & $? / \mathrm{B}$ & $+/ \mathrm{D}$ & $0^{\mathrm{n}}$ & 0 & 0 & 0 & $\pm / \mathrm{B}$ & 0 \\
\hline $\begin{array}{l}\text { Immunosuppressant } \\
\text { Therapy Barrier Scale }\end{array}$ & 1 & $\mathrm{C}$ & $+/ \mathrm{B}$ & $? / \mathrm{B}$ & $+/ \mathrm{A}$ & 0 & 0 & 0 & 0 & $+/ \mathrm{A}$ & $+/ \mathrm{A}$ \\
\hline Kennedy et $\mathrm{al}^{\mathrm{m}}$ [44] & 1 & $\mathrm{D}$ & $+/ \mathrm{B}$ & 0 & 0 & 0 & 0 & 0 & 0 & 0 & 0 \\
\hline Kerr et $\mathrm{al}^{\mathrm{m}}$ [45] & 1 & $\mathrm{D}$ & 0 & 0 & 0 & 0 & 0 & 0 & 0 & $-/ \mathrm{D}$ & $-/ \mathrm{D}$ \\
\hline $\begin{array}{l}\text { Least absolute shrink- } \\
\text { age and selection opera- } \\
\text { tor-10 }\end{array}$ & 1 & B & $+/ \mathrm{B}$ & ?/B & 0 & 0 & 0 & 0 & 0 & $? / \mathrm{C}$ & $+/ \mathrm{B}$ \\
\hline $\begin{array}{l}\text { Long-Term Medication } \\
\text { Behaviour Self-Effica- } \\
\text { cy Scale }\end{array}$ & 1 & $\mathrm{D}$ & ?/D & 0 & 0 & 0 & 0 & 0 & 0 & 0 & 0 \\
\hline $\begin{array}{l}\text { Modified Drug Adher- } \\
\text { ence Work-up Tool }\end{array}$ & 2 & 0 & ?/D & $? / \mathrm{D}$ & $+/ \mathrm{D}$ & 0 & 0 & 0 & 0 & $-/ \mathrm{B}$ & $+/ \mathrm{B}$ \\
\hline $\begin{array}{l}\text { Medication Adherence } \\
\text { Questionnaire }\end{array}$ & 1 & $\mathrm{D}$ & ?/D & 0 & $+/ \mathrm{D}$ & $0^{\mathrm{n}}$ & $+/ \mathrm{D}$ & 0 & 0 & 0 & 0 \\
\hline MAR-Scale ${ }^{r}$ & 1 & $\mathrm{C}$ & $? / \mathrm{D}$ & ?/B & $-/ \mathrm{A}$ & 0 & $-/ \mathrm{D}$ & 0 & 0 & $+/ \mathrm{D}$ & 0 \\
\hline MAR-Scale (revised) & 2 & B & $+/ \mathrm{B}$ & $? / \mathrm{B}$ & $+/ \mathrm{A}$ & 0 & 0 & 0 & 0 & $+/ \mathrm{B}$ & 0 \\
\hline MARS $S^{S}$ & 8 & $\mathrm{D}$ & $+/ \mathrm{D}$ & ?/B & $\pm / \mathrm{A}$ & $0^{\mathrm{n}}$ & $? / \mathrm{D}$ & 0 & 0 & $\pm / \mathrm{B}$ & $\pm / \mathrm{B}$ \\
\hline MARS-10 & 1 & 0 & ?/D & ?/B & $+/ \mathrm{A}$ & $0^{\mathrm{n}}$ & 0 & 0 & 0 & $+/ \mathrm{B}$ & $+/ \mathrm{B}$ \\
\hline MARS-5 & 8 & 0 & $+/ \mathrm{B}$ & ?/B & $+/ \mathrm{B}$ & $0^{\mathrm{n}}$ & $\pm / \mathrm{B}$ & 0 & 0 & $\pm / \mathrm{A}$ & $\pm / \mathrm{B}$ \\
\hline MARS-9 & 2 & 0 & $+/ \mathrm{B}$ & ?/B & $+/ \mathrm{A}$ & 0 & $-/ \mathrm{B}$ & 0 & 0 & $\pm / \mathrm{B}$ & 0 \\
\hline MASES $^{t}$ & 2 & $\mathrm{D}$ & $+/ \mathrm{A}$ & $? / \mathrm{B}$ & $+/ \mathrm{A}$ & 0 & $-/ \mathrm{D}$ & 0 & 0 & $\pm / \mathrm{A}$ & 0 \\
\hline MASES-R & 1 & 0 & 0 & ?/B & $+/ \mathrm{D}$ & 0 & $-/ \mathrm{C}$ & 0 & 0 & $-/ \mathrm{B}$ & 0 \\
\hline $\begin{array}{l}\text { Medication Adherence } \\
\text { Self-Report Inventory }\end{array}$ & 6 & $\mathrm{D}$ & 0 & 0 & $+/ \mathrm{B}$ & 0 & $+/ \mathrm{D}$ & 0 & 0 & $+/ \mathrm{A}$ & $+/ \mathrm{A}$ \\
\hline $\begin{array}{l}\text { Medication adherence } \\
\text { scale }\end{array}$ & 1 & $\mathrm{D}$ & $+/ \mathrm{C}$ & $-/ \mathrm{A}$ & $+/ \mathrm{A}$ & 0 & 0 & 0 & 0 & $-/ \mathrm{B}$ & 0 \\
\hline $\begin{array}{l}\text { Medication adherence } \\
\text { survey }\end{array}$ & 1 & B & $+/ \mathrm{B}$ & 0 & 0 & 0 & 0 & 0 & 0 & $+/ \mathrm{D}$ & 0 \\
\hline $\begin{array}{l}\text { Medication Adherence } \\
\text { Estimation and Differen- } \\
\text { tiation Scale }\end{array}$ & 1 & $\mathrm{C}$ & ?/B & $+/ \mathrm{A}$ & $+/ \mathrm{A}$ & 0 & 0 & 0 & 0 & $+/ \mathrm{D}$ & $-/ \mathrm{A}$ \\
\hline
\end{tabular}




\begin{tabular}{|c|c|c|c|c|c|c|c|c|c|c|c|}
\hline $\mathrm{PROM}^{\mathrm{a}}$ & $\begin{array}{l}\text { Num- } \\
\text { ber of } \\
\text { studies }\end{array}$ & $\begin{array}{l}\text { PROM } \\
\text { develop- } \\
\text { ment }\end{array}$ & $\begin{array}{l}\text { Con- } \\
\text { tent } \\
\text { validi- } \\
\text { ty }\end{array}$ & $\begin{array}{l}\text { Struc- } \\
\text { tural va- } \\
\text { lidity }\end{array}$ & $\begin{array}{l}\text { Internal } \\
\text { consisten- } \\
\text { cy }\end{array}$ & $\begin{array}{l}\text { Cross-cultur- } \\
\text { al validi- } \\
\text { ty/measure- } \\
\text { ment invari- } \\
\text { ance }\end{array}$ & $\begin{array}{l}\text { Reliabil- } \\
\text { ity }\end{array}$ & $\begin{array}{l}\text { Mea- } \\
\text { sure- } \\
\text { ment er- } \\
\text { ror }\end{array}$ & $\begin{array}{l}\text { Criteri- } \\
\text { on valid- } \\
\text { ity }\end{array}$ & $\begin{array}{l}\text { Hypothe- } \\
\text { ses test- } \\
\text { ing for } \\
\text { construct } \\
\text { validity }\end{array}$ & $\begin{array}{l}\text { Responsive- } \\
\text { ness }\end{array}$ \\
\hline $\begin{array}{l}\text { Medication Intake Sur- } \\
\text { vey-Asthma }\end{array}$ & 1 & B & $+/ \mathrm{B}$ & 0 & 0 & 0 & $-/ \mathrm{B}$ & 0 & 0 & $+/ \mathrm{B}$ & 0 \\
\hline MMAS $^{\mathrm{u}}-4$ & 18 & 0 & $+/ \mathrm{C}$ & ?/B & $-/ \mathrm{B}$ & 0 & $\pm / \mathrm{B}$ & 0 & 0 & $\pm / \mathrm{A}$ & $-/ \mathrm{B}$ \\
\hline MMAS-7 & 1 & 0 & 0 & 0 & 0 & $0^{\mathrm{n}}$ & 0 & 0 & 0 & $+/ \mathrm{B}$ & 0 \\
\hline MMAS-8 & 27 & 0 & $+/ \mathrm{B}$ & $\pm / \mathrm{A}$ & $\pm / \mathrm{A}$ & $0^{\mathrm{n}}$ & $+/ \mathrm{B}$ & 0 & 0 & $\pm / \mathrm{A}$ & $-/ \mathrm{B}$ \\
\hline MMAS-9 & 1 & 0 & 0 & $+/ \mathrm{A}$ & $-/ \mathrm{A}$ & 0 & $? / \mathrm{C}$ & 0 & 0 & $-/ \mathrm{B}$ & 0 \\
\hline $\begin{array}{l}\text { Medication Nonpersis- } \\
\text { tence Scale }\end{array}$ & 1 & $\mathrm{C}$ & $? / \mathrm{C}$ & $+/ \mathrm{A}$ & $+/ \mathrm{D}$ & 0 & 0 & 0 & 0 & $+/ \mathrm{C}$ & $-/ \mathrm{D}$ \\
\hline $\begin{array}{l}\text { Medical Outcomes } \\
\text { Study General Adher- } \\
\text { ence Scale }\end{array}$ & 1 & 0 & 0 & 0 & $+/ \mathrm{D}$ & 0 & 0 & 0 & 0 & $-/ \mathrm{C}$ & 0 \\
\hline $\begin{array}{l}\text { Multiple Sclerosis } \\
\text { Treatment Adherence } \\
\text { Questionnaire }\end{array}$ & 1 & $\mathrm{C}$ & ?/B & 0 & $-/ \mathrm{A}$ & 0 & 0 & 0 & 0 & $+/ \mathrm{A}$ & 0 \\
\hline $\begin{array}{l}\text { Outcome Expectations } \\
\text { for Osteoporosis Medi- } \\
\text { cation Adherence Scale }\end{array}$ & 2 & $\mathrm{D}$ & $+/ \mathrm{B}$ & $\pm / \mathrm{A}$ & $+/ \mathrm{A}$ & 0 & 0 & 0 & 0 & $-/ \mathrm{C}$ & 0 \\
\hline $\begin{array}{l}\text { Perceived Barriers to } \\
\text { Antiretroviral Therapy } \\
\text { Adherence Scale }\end{array}$ & 1 & B & $+/ \mathrm{C}$ & ?/B & $? / \mathrm{C}$ & $0^{\mathrm{n}}$ & $-/ \mathrm{C}$ & 0 & 0 & $+/ \mathrm{C}$ & 0 \\
\hline $\begin{array}{l}\text { Pictographic Self-Effica- } \\
\text { cy Scale }\end{array}$ & 1 & $\mathrm{D}$ & $+/ \mathrm{B}$ & 0 & $-/ \mathrm{A}$ & 0 & $-/ \mathrm{D}$ & 0 & 0 & $-/ \mathrm{B}$ & 0 \\
\hline $\begin{array}{l}\text { Patient Rating of Com- } \\
\text { pliance Scale }\end{array}$ & 1 & 0 & 0 & 0 & 0 & 0 & 0 & 0 & 0 & $+/ \mathrm{B}$ & 0 \\
\hline $\begin{array}{l}\text { Patient Preference } \\
\text { Questionnaire }\end{array}$ & 1 & $\mathrm{D}$ & $+/ \mathrm{D}$ & 0 & $+/ \mathrm{D}$ & 0 & 0 & 0 & 0 & 0 & 0 \\
\hline $\begin{array}{l}\text { Probabilistic Medica- } \\
\text { tion Adherence Scale }\end{array}$ & 1 & $\mathrm{C}$ & $\pm / \mathrm{C}$ & $-/ \mathrm{A}$ & $+/ \mathrm{C}$ & 0 & 0 & 0 & 0 & 0 & 0 \\
\hline $\begin{array}{l}\text { Number of pills taken } \\
\text { or prescribed }\end{array}$ & 2 & 0 & 0 & 0 & $? / \mathrm{D}$ & 0 & $-/ \mathrm{D}$ & 0 & 0 & $\pm / \mathrm{C}$ & 0 \\
\hline $\begin{array}{l}\text { Questionnaire for Ad- } \\
\text { herence with Topical } \\
\text { Treatments in Psoriasis }\end{array}$ & 1 & $\mathrm{D}$ & $+/ \mathrm{B}$ & 0 & 0 & 0 & 0 & 0 & 0 & $\pm / \mathrm{D}$ & 0 \\
\hline Question of Interest & 1 & 0 & $? / \mathrm{D}$ & 0 & 0 & 0 & 0 & 0 & 0 & $-/ \mathrm{D}$ & $-/ \mathrm{D}$ \\
\hline $\mathrm{SCI}^{\mathrm{V}}$ & 2 & 0 & $? / \mathrm{C}$ & $+/ \mathrm{A}$ & 0 & 0 & $-/ \mathrm{B}$ & 0 & 0 & $\pm / \mathrm{A}$ & $-/ \mathrm{B}$ \\
\hline SCI-R & 1 & 0 & $+/ \mathrm{B}$ & $? / \mathrm{B}$ & $+/ \mathrm{A}$ & $0^{\mathrm{n}}$ & $? / \mathrm{D}$ & 0 & 0 & $+/ \mathrm{D}$ & $+/ \mathrm{D}$ \\
\hline $\begin{array}{l}\text { Strathclyde Compliance } \\
\text { Risk Assessment Tool }\end{array}$ & 2 & 0 & 0 & 0 & $+/ \mathrm{B}$ & 0 & 0 & 0 & 0 & $+/ \mathrm{B}$ & $+/ \mathrm{B}$ \\
\hline $\begin{array}{l}\text { Summary of Diabetes } \\
\text { Self-care Activities }\end{array}$ & 2 & 0 & 0 & 0 & 0 & 0 & 0 & 0 & 0 & $\pm / \mathrm{C}$ & 0 \\
\hline $\begin{array}{l}\text { Self-Efficacy for Appro- } \\
\text { priate Medication Ad- } \\
\text { herence Scale }\end{array}$ & 3 & $\mathrm{~B}$ & $+/ \mathrm{B}$ & ?/B & $+/ \mathrm{A}$ & 0 & $+/ \mathrm{A}$ & 0 & 0 & $\pm / \mathrm{A}$ & 0 \\
\hline Self-efficacy scale & 1 & $\mathrm{D}$ & ?/B & $? / \mathrm{B}$ & $+/ \mathrm{A}$ & 0 & 0 & 0 & 0 & $? / \mathrm{D}$ & 0 \\
\hline $\begin{array}{l}\text { Self-report measures of } \\
\text { adherence }\end{array}$ & 1 & 0 & 0 & 0 & 0 & 0 & ?/B & 0 & 0 & $+/ \mathrm{B}$ & 0 \\
\hline $\begin{array}{l}\text { Self-report on adher- } \\
\text { ence }\end{array}$ & 1 & 0 & 0 & 0 & 0 & 0 & 0 & 0 & 0 & $-/ \mathrm{C}$ & $+/ \mathrm{C}$ \\
\hline
\end{tabular}




\begin{tabular}{|c|c|c|c|c|c|c|c|c|c|c|c|}
\hline $\mathrm{PROM}^{\mathrm{a}}$ & $\begin{array}{l}\text { Num- } \\
\text { ber of } \\
\text { studies }\end{array}$ & $\begin{array}{l}\text { PROM } \\
\text { develop- } \\
\text { ment }\end{array}$ & $\begin{array}{l}\text { Con- } \\
\text { tent } \\
\text { validi- } \\
\text { ty }\end{array}$ & $\begin{array}{l}\text { Struc- } \\
\text { tural va- } \\
\text { lidity }\end{array}$ & $\begin{array}{l}\text { Internal } \\
\text { consisten- } \\
\text { cy }\end{array}$ & $\begin{array}{l}\text { Cross-cultur- } \\
\text { al validi- } \\
\text { ty/measure- } \\
\text { ment invari- } \\
\text { ance }\end{array}$ & $\begin{array}{l}\text { Reliabil- } \\
\text { ity }\end{array}$ & $\begin{array}{l}\text { Mea- } \\
\text { sure- } \\
\text { ment er- } \\
\text { ror }\end{array}$ & $\begin{array}{l}\text { Criteri- } \\
\text { on valid- } \\
\text { ity }\end{array}$ & $\begin{array}{l}\text { Hypothe- } \\
\text { ses test- } \\
\text { ing for } \\
\text { construct } \\
\text { validity }\end{array}$ & $\begin{array}{l}\text { Responsive- } \\
\text { ness }\end{array}$ \\
\hline $\begin{array}{l}\text { Self-Efficacy for Osteo- } \\
\text { porosis Medication Ad- } \\
\text { herence Scale }\end{array}$ & 2 & $\mathrm{D}$ & $+/ \mathrm{B}$ & $-/ \mathrm{A}$ & $+/ \mathrm{A}$ & 0 & 0 & 0 & 0 & $+/ \mathrm{C}$ & 0 \\
\hline $\begin{array}{l}\text { Self-Reported Adher- } \\
\text { ence Questionnaire }\end{array}$ & 1 & 0 & $\mathrm{D}$ & 0 & 0 & 0 & 0 & 0 & 0 & $? / \mathrm{D}$ & 0 \\
\hline $\begin{array}{l}\text { Satisfaction with Iron } \\
\text { Chelation Therapy }\end{array}$ & 1 & $\mathrm{~B}$ & $+/ \mathrm{B}$ & ?/B & $+/ \mathrm{A}$ & 0 & 0 & 0 & 0 & $+/ \mathrm{A}$ & 0 \\
\hline $\begin{array}{l}\text { Sidorkiewicz et al }{ }^{\mathrm{m}} \\
\text { [46] }\end{array}$ & 1 & $\mathrm{~B}$ & $+/ \mathrm{B}$ & 0 & 0 & 0 & 0 & 0 & 0 & $+/ \mathrm{C}$ & 0 \\
\hline $\begin{array}{l}\text { Simplified Medication } \\
\text { Adherence Question- } \\
\text { naire }\end{array}$ & 2 & $\mathrm{D}$ & $? / \mathrm{C}$ & 0 & $+/ \mathrm{C}$ & 0 & $+/ \mathrm{A}$ & 0 & 0 & $+/ \mathrm{B}$ & $-/ \mathrm{A}$ \\
\hline $\begin{array}{l}\text { Stages of Change Mod- } \\
\text { el Questionnaire }\end{array}$ & 2 & $\mathrm{D}$ & 0 & 0 & 0 & 0 & 0 & 0 & 0 & $+/ \mathrm{C}$ & 0 \\
\hline $\begin{array}{l}\text { Special Projects of Na- } \\
\text { tional Significance Ad- } \\
\text { herence Survey }\end{array}$ & 1 & 0 & 0 & 0 & $+/ \mathrm{D}$ & 0 & 0 & 0 & 0 & $+/ \mathrm{C}$ & 0 \\
\hline Self-Rating Scale Item & 4 & 0 & 0 & 0 & 0 & 0 & 0 & 0 & 0 & $+/ \mathrm{C}$ & $+/ \mathrm{C}$ \\
\hline $\begin{array}{l}\text { Test of Adherence to } \\
\text { Inhalers }\end{array}$ & 1 & B & ?/B & ?/B & $+/ \mathrm{D}$ & 0 & $+/ \mathrm{A}$ & 0 & 0 & $-/ \mathrm{C}$ & $-/ \mathrm{A}$ \\
\hline Tan et al [47] & 1 & $\mathrm{C}$ & $? / \mathrm{C}$ & ?/B & $+/ \mathrm{A}$ & 0 & $+/ \mathrm{A}$ & 0 & 0 & $+/ \mathrm{B}$ & 0 \\
\hline $\begin{array}{l}\text { Treatment Adherence } \\
\text { Survey-Patient Version }\end{array}$ & 1 & $\mathrm{D}$ & 0 & 0 & 0 & 0 & $+/ \mathrm{B}$ & 0 & 0 & $-/ \mathrm{C}$ & 0 \\
\hline $\begin{array}{l}\text { Therapeutic Adherence } \\
\text { Scale for Hypertensive } \\
\text { Patients }\end{array}$ & 1 & 0 & 0 & $+/ \mathrm{A}$ & $+/ \mathrm{A}$ & 0 & 0 & 0 & 0 & $+/ \mathrm{B}$ & $-/ \mathrm{B}$ \\
\hline $\begin{array}{l}\text { Topical Therapy Adher- } \\
\text { ence Questionnaire }\end{array}$ & 1 & $\mathrm{D}$ & $+/ \mathrm{D}$ & 0 & $+/ \mathrm{D}$ & 0 & 0 & 0 & 0 & 0 & 0 \\
\hline $\begin{array}{l}\text { Turcu- tiolică et al }{ }^{\mathrm{m}} \\
\text { [48] }\end{array}$ & 1 & $\mathrm{D}$ & $+/ \mathrm{D}$ & 0 & $-/ \mathrm{D}$ & 0 & 0 & 0 & 0 & $-/ \mathrm{D}$ & 0 \\
\hline Visual analog scale & 4 & 0 & 0 & 0 & 0 & 0 & $-/ \mathrm{D}$ & 0 & 0 & $+/ \mathrm{B}$ & 0 \\
\hline $\begin{array}{l}\text { Validated Hemophilia } \\
\text { Regimen Treatment } \\
\text { Adherence Scale-On- } \\
\text { Demand }\end{array}$ & 1 & $\mathrm{D}$ & $+/ \mathrm{D}$ & 0 & $+/ \mathrm{B}$ & 0 & $+/ \mathrm{B}$ & 0 & 0 & $\pm / \mathrm{B}$ & 0 \\
\hline $\begin{array}{l}\text { Validated Hemophilia } \\
\text { Regimen Treatment } \\
\text { Regimen Treatment } \\
\text { Adherence Scale-Pro- } \\
\text { phylaxis }\end{array}$ & 1 & $\mathrm{D}$ & $+/ \mathrm{D}$ & 0 & $+/ \mathrm{C}$ & 0 & $+/ \mathrm{C}$ & 0 & 0 & $-/ \mathrm{C}$ & 0 \\
\hline Voils et $\mathrm{al}^{\mathrm{m}}$ [49] & 4 & $\mathrm{C}$ & $+/ \mathrm{B}$ & $+/ \mathrm{A}$ & $+/ \mathrm{A}$ & $0^{\mathrm{n}}$ & $-/ \mathrm{A}$ & 0 & 0 & $\pm / \mathrm{A}$ & $-/ \mathrm{B}$ \\
\hline Vreeman et $\mathrm{al}^{\mathrm{m}}[50]$ & 2 & $\mathrm{C}$ & $? / \mathrm{C}$ & 0 & 0 & $0^{\mathrm{n}}$ & 0 & 0 & 0 & $-/ \mathrm{C}$ & $-/ \mathrm{C}$ \\
\hline $\begin{array}{l}\text { Web-Ad-Q Question- } \\
\text { naire }\end{array}$ & 1 & $\mathrm{C}$ & $+/ \mathrm{C}$ & 0 & 0 & 0 & $+/ \mathrm{D}$ & 0 & 0 & $+/ \mathrm{C}$ & 0 \\
\hline
\end{tabular}




\begin{tabular}{|c|c|c|c|c|c|c|c|c|c|c|c|}
\hline $\mathrm{PROM}^{\mathrm{a}}$ & $\begin{array}{l}\text { Num- } \\
\text { ber of } \\
\text { studies }\end{array}$ & $\begin{array}{l}\text { PROM } \\
\text { develop- } \\
\text { ment }\end{array}$ & $\begin{array}{l}\text { Con- } \\
\text { tent } \\
\text { validi- } \\
\text { ty }\end{array}$ & $\begin{array}{l}\text { Struc- } \\
\text { tural va- } \\
\text { lidity }\end{array}$ & $\begin{array}{l}\text { Internal } \\
\text { consisten- } \\
\text { cy }\end{array}$ & $\begin{array}{l}\text { Cross-cultur- } \\
\text { al validi- } \\
\text { ty/measure- } \\
\text { ment invari- } \\
\text { ance }\end{array}$ & $\begin{array}{l}\text { Reliabil- } \\
\text { ity }\end{array}$ & $\begin{array}{l}\text { Mea- } \\
\text { sure- } \\
\text { ment er- } \\
\text { ror }\end{array}$ & $\begin{array}{l}\text { Criteri- } \\
\text { on valid- } \\
\text { ity }\end{array}$ & $\begin{array}{l}\text { Hypothe- } \\
\text { ses test- } \\
\text { ing for } \\
\text { construct } \\
\text { validity }\end{array}$ & $\begin{array}{l}\text { Responsive- } \\
\text { ness }\end{array}$ \\
\hline Wilson et $\mathrm{al}^{\mathrm{m}}$ [51] & 3 & B & $+/ \mathrm{B}$ & 0 & $+/ \mathrm{A}$ & 0 & 0 & 0 & 0 & $+/ \mathrm{D}$ & $-/ \mathrm{A}$ \\
\hline
\end{tabular}

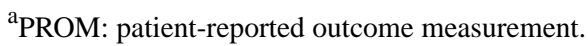

${ }^{\mathrm{b}} 0$ : Measurement properties were not assessed by the study.

${ }^{c_{\text {? }} \text { : intermediate. }}$

${ }^{\mathrm{d}} \mathrm{B}$ : moderate.

e-: insufficient.

${ }^{\mathrm{f}} \mathrm{C}$ : low.

$\mathrm{g}_{+}$: sufficient.

${ }^{\mathrm{h}} \mathrm{D}$ : very low.

${ }^{\mathrm{i}} \mathrm{A}$ : high.

$\mathrm{j}_{ \pm:}$inconsistent.

${ }^{\mathrm{k}}$ ARMS: Adherence to Refills and Medications Scale.

${ }^{1}$ ASK: Adherence Starts with Knowledge questionnaire.

${ }^{m}$ PROMs without proper names are labeled based on the last name of the first author who developed the instrument.

${ }^{\mathrm{n}}$ Only translation was done. Cross-cultural validation was not the aim of the study.

${ }^{\circ} \mathrm{CQR}$ : Compliance Questionnaire on Rheumatology.

${ }^{\mathrm{p}}$ DAI: Drug Attitude Inventory.

${ }^{\mathrm{q}}$ GTCAT: Glaucoma Treatment Compliance Assessment Tool.

${ }^{\mathrm{r}}$ MAR-Scale: Medication Adherence Reasons Scale.

${ }^{\mathrm{s}}$ MARS: Medication Adherence Rating Scale.

${ }^{t}$ MASES: Medication Adherence Self-efficacy Scale.

uMMAS: Morisky Medication Adherence Scale.

${ }^{\mathrm{v}} \mathrm{SCI}$ : Self-Care Inventory.

\section{Discussion}

To the best of our knowledge, this is the first systematic review that comprehensively summarized PROMs for medication adherence based on the COSMIN guidelines [21,22]. Among the 214 included articles, we identified 121 unique PROMs for medication adherence. Our study revealed the most commonly evaluated medication adherence PROMs to be the MMAS-8, MMAS-4, AACTG, MARS, and MARS-5. However, being more commonly evaluated does not mean that these PROMs have the best psychometric properties. Moreover, based on the number of studies for each PROM in Table 2, most of the PROMs have too few studies to provide a strong evidence base for their use.

Among the 15 PROMs that have been administered electronically, 3 PROMs (MAR-Scale revised, MMAS-4, and MMAS-8) have at least a moderate level of evidence for $\geq 5$ measurement properties. Electronic administration of PROMs to measure medication adherence may be appealing in health care settings, as it may reduce the administrative burden for data collection and data entry. In addition, as web-based interventions to improve medication adherence become increasingly commonplace [250], electronic PROMs may be incorporated into web-based platforms to assess the effectiveness of these web-based interventions.
Despite a few studies claiming the use of certain PROMs and objective measures as the gold standard for measuring medication adherence, we deliberately omitted evaluating criterion validity for these studies. As mentioned in the introduction, although objective measures such as pill count, electronic monitoring devices, and big data may measure adherence indirectly, these measures are laborious, costly, and sometimes invasive, making them unsuitable for routine clinical use. Furthermore, all these surrogate measures, including PROMs, do not predict any real biological outcomes such as a reduction in viral load, blood pressure, or glucose concentration in determining medication adherence in patients. Hence, none of these measures can be deemed as a gold standard [10].

Measurement error was not evaluated because none of the studies reported the standard error of measurement, smallest detectable change, or limits of agreements required by the COSMIN. In addition, although translations of PROMs were performed in 46 studies, none of these studies assessed measurement invariance or differential item functioning; therefore, cross-cultural validity was not evaluated for any of the PROMs in this study. Moreover, only one study examined the interpretability of PROMs in the form of minimal detectable change for the MMAS-8 [251]. Further study on measurement error, cross-cultural validity, and interpretability of medication adherence PROMs is warranted.

The strengths of this study include using COSMIN guidelines, which are well regarded as a consensus-based standard for 
evaluating the measurement properties of PROMs [23]. The COSMIN Risk of Bias checklist employed in this study is an improvement from the original COSMIN checklist with several improvements in the standards for evaluation $[21,22]$. We also used sensitive search filters to retrieve and include as many potentially relevant articles as possible.

One limitation related to this study was that the selection of articles and evaluation of psychometric properties were subjective in nature and may have been prone to judgment bias. Nevertheless, the requirement by COSMIN to have 2 independent reviewers and the need for a third reviewer to reach a consensus in the case of any discrepancy occurring has helped reduce the risk of judgment bias [22,252].

\section{Conclusions}

In summary, 121 unique medication adherence PROMs were identified in 214 studies. On the basis of the COSMIN guidelines, PROMs with at least a moderate level of evidence for $\geq 5$ measurement properties include the ASK-20, CQR, GMAS, Hill-Bone Scale, ITBS, MAR-Scale revised, MARS-5, MARS-9, MMAS-4, MMAS-8, SEAMS, SICT, TAI, and questionnaire by Voils. Of these, only the GMAS has sufficient $(+)$ ratings for at least four measurement properties. We believe this study would assist clinicians and researchers in selecting suitable PROMs to measure medication adherence among patients. Future research may consider validating measurement errors and cross-cultural validity to further improve the insights on the measurement properties of these PROMs.

\section{Acknowledgments}

The authors would like to thank Librarian Ms Wong Suei Nee at the National University of Singapore Medical Library for her advice on search strategies. This research was supported by the SingHealth Regional Health System Population-based, Unified, Learning System for Enhanced and Sustainable Health (PULSES) Centre Grant-Measuring Medication Adherence in Singapore (CGDec19S03), and PULSES Fellowship (CGDec19F02).

Kwan Yu Heng, Weng Si Dun, and Dionne Hui Fang Loh contributed equally as co-first authors. Truls Østbye, Low Lian Leng, Hayden Barry Bosworth, and Julian Thumboo contributed equally as senior coauthors.

\section{Conflicts of Interest}

None declared.

\section{Multimedia Appendix 1}

Search strategy.

[DOCX File, 33 KB-Multimedia Appendix 1]

\section{Multimedia Appendix 2}

Characteristics of patient reported outcome measures.

[DOCX File , 45 KB-Multimedia Appendix 2]

\section{Multimedia Appendix 3}

Assessment of psychometric properties of patient-reported outcome measures.

[DOCX File , 64 KB-Multimedia Appendix 3]

\section{References}

1. Dal-Fabbro AL. Adherence to long term therapies: evidence for action. Cad. Saúde Pública 2005 Aug;21(4):1297-1298. [doi: 10.1590/s0102-311x2005000400037]

2. Asche $\mathrm{C}$, LaFleur J, Conner C. A review of diabetes treatment adherence and the association with clinical and economic outcomes. Clin Ther 2011 Jan;33(1):74-109. [doi: 10.1016/j.clinthera.2011.01.019] [Medline: 21397776]

3. Han E, Suh D, Lee S, Jang S. The impact of medication adherence on health outcomes for chronic metabolic diseases: a retrospective cohort study. Res Social Adm Pharm 2014;10(6):e87-e98. [doi: 10.1016/j.sapharm.2014.02.001] [Medline: 25088545]

4. Sabaté E. editor., ed. Adherence to Long-Term Therapiesvidence for Action. Geneva, Switzerland: World Health Organization; 2003:E.

5. Lee S, Raamkumar AS, Li J, Cao Y, Witedwittayanusat K, Chen L, et al. Reasons for primary medication nonadherence: a systematic review and metric analysis. J Manag Care Spec Pharm 2018 Aug;24(8):778-794 [FREE Full text] [doi: 10.18553/jmcp.2018.24.8.778] [Medline: 30058985]

6. Ho PM, Rumsfeld JS, Masoudi FA, McClure DL, Plomondon ME, Steiner JF, et al. Effect of medication nonadherence on hospitalization and mortality among patients with diabetes mellitus. Arch Intern Med 2006 Sep 25;166(17):1836-1841. [doi: 10.1001/archinte.166.17.1836] [Medline: 17000939] 
7. Haynes RB, Ackloo E, Sahota N, McDonald HP, Yao X. Interventions for enhancing medication adherence. Cochrane Database Syst Rev 2008 Apr 16(2):CD000011. [doi: 10.1002/14651858.CD000011.pub3] [Medline: 18425859]

8. Brown M, Bussell J. Medication adherence: WHO cares? Mayo Clin Proc 2011 Apr;86(4):304-314 [FREE Full text] [doi: 10.4065/mcp.2010.0575] [Medline: 21389250]

9. Zhang JX, Meltzer DO. Identifying patients with cost-related medication non-adherence: a big-data approach. J Med Econ 2016 Aug;19(8):806-811 [FREE Full text] [doi: 10.1080/13696998.2016.1176031] [Medline: 27052465]

10. Lam WY, Fresco P. Medication adherence measures: an overview. Biomed Res Int 2015;2015:217047 [FREE Full text] [doi: 10.1155/2015/217047] [Medline: 26539470]

11. Moon SJ, Lee W, Hwang JS, Hong YP, Morisky DE. Accuracy of a screening tool for medication adherence: a systematic review and meta-analysis of the Morisky medication adherence scale-8. PLoS One 2017;12(11):e0187139 [FREE Full text] [doi: 10.1371/journal.pone.0187139] [Medline: 29095870]

12. Kim M, Hill M, Bone L, Levine D. Development and testing of the hill-bone compliance to high blood pressure therapy scale. Prog Cardiovasc Nurs 2000;15(3):90-96. [doi: 10.1111/j.1751-7117.2000.tb00211.x] [Medline: 10951950]

13. Thompson K, Kulkarni J, Sergejew A. Reliability and validity of a new medication adherence rating scale (MARS) for the psychoses. Schizophr Res 2000 May 5;42(3):241-247. [doi: 10.1016/s0920-9964(99)00130-9] [Medline: 10785582]

14. Voils CI, Hoyle RH, Thorpe CT, Maciejewski ML, Yancy WS. Improving the measurement of self-reported medication nonadherence. J Clin Epidemiol 2011 Mar;64(3):250-254. [doi: 10.1016/j.jclinepi.2010.07.014] [Medline: 21194887]

15. Voils CI, Maciejewski ML, Hoyle RH, Reeve BB, Gallagher P, Bryson CL, et al. Initial validation of a self-report measure of the extent of and reasons for medication nonadherence. Med Care 2012 Dec;50(12):1013-1019 [FREE Full text] [doi: 10.1097/MLR.0b013e318269e121] [Medline: 22922431]

16. Korb-Savoldelli V, Gillaizeau F, Pouchot J, Lenain E, Postel-Vinay N, Plouin PF, et al. Validation of a French version of the 8-item Morisky medication adherence scale in hypertensive adults. J Clin Hypertens (Greenwich) 2012 Jul;14(7):429-434 [FREE Full text] [doi: 10.1111/j.1751-7176.2012.00634.x] [Medline: 22747615]

17. Fialko L, Garety PA, Kuipers E, Dunn G, Bebbington PE, Fowler D, et al. A large-scale validation study of the medication adherence rating scale (MARS). Schizophr Res 2008 Mar;100(1-3):53-59. [doi: 10.1016/j.schres.2007.10.029] [Medline: $\underline{18083007]}$

18. Gagné M, Boulet L, Pérez N, Moisan J. Patient-reported outcome instruments that evaluate adherence behaviours in adults with asthma: a systematic review of measurement properties. Br J Clin Pharmacol 2018 Sep;84(9):1928-1940 [FREE Full text] [doi: 10.1111/bcp.13623] [Medline: 29710423]

19. Moher D, Liberati A, Tetzlaff J, Altman DG, PRISMA Group. Preferred reporting items for systematic reviews and meta-analyses: the PRISMA statement. PLoS Med 2009 Jul 21;6(7):e1000097 [FREE Full text] [doi: 10.1371/journal.pmed.1000097] [Medline: 19621072]

20. Mokkink LB, Terwee CB, Patrick DL, Alonso J, Stratford PW, Knol DL, et al. The COSMIN checklist for assessing the methodological quality of studies on measurement properties of health status measurement instruments: an international Delphi study. Qual Life Res 2010 May;19(4):539-549 [FREE Full text] [doi: 10.1007/s11136-010-9606-8] [Medline: 20169472]

21. Mokkink LB, de Vet HC, Prinsen CA, Patrick DL, Alonso J, Bouter LM, et al. COSMIN risk of bias checklist for systematic reviews of patient-reported outcome measures. Qual Life Res 2018 May;27(5):1171-1179 [FREE Full text] [doi: 10.1007/s11136-017-1765-4] [Medline: 29260445]

22. Prinsen CAC, Mokkink LB, Bouter LM, Alonso J, Patrick DL, de Vet HC, et al. COSMIN guideline for systematic reviews of patient-reported outcome measures. Qual Life Res 2018 May;27(5):1147-1157 [FREE Full text] [doi: 10.1007/s11136-018-1798-3] [Medline: 29435801]

23. Mokkink LB, Prinsen CA, Bouter LM, Vet HC, Terwee CB. The consensus-based standards for the selection of health measurement instruments (COSMIN) and how to select an outcome measurement instrument. Braz J Phys Ther 2016 Jan 19;20(2):105-113 [FREE Full text] [doi: 10.1590/bjpt-rbf.2014.0143] [Medline: 26786084]

24. Mokkink LB, Terwee CB, Patrick DL, Alonso J, Stratford PW, Knol DL, et al. The COSMIN study reached international consensus on taxonomy, terminology, and definitions of measurement properties for health-related patient-reported outcomes. J Clin Epidemiol 2010 Jul;63(7):737-745. [doi: 10.1016/j.jclinepi.2010.02.006] [Medline: 20494804]

25. Davidson M, Keating J. Patient-reported outcome measures (PROMs): how should I interpret reports of measurement properties? A practical guide for clinicians and researchers who are not biostatisticians. Br J Sports Med 2014 May;48(9):792-796. [doi: 10.1136/bjsports-2012-091704] [Medline: 23258849]

26. Husted JA, Cook RJ, Farewell VT, Gladman DD. Methods for assessing responsiveness: a critical review and recommendations. J Clin Epidemiol 2000 May;53(5):459-468. [doi: 10.1016/s0895-4356(99)00206-1] [Medline: 10812317]

27. Terwee CB, Jansma EP, Riphagen II, de Vet HC. Development of a methodological PubMed search filter for finding studies on measurement properties of measurement instruments. Qual Life Res 2009 Oct;18(8):1115-1123 [FREE Full text] [doi: 10.1007/s11136-009-9528-5] [Medline: 19711195]

28. Terwee CB, Prinsen CA, Ricci Garotti MG, Suman A, de Vet HC, Mokkink LB. The quality of systematic reviews of health-related outcome measurement instruments. Qual Life Res 2016 Apr;25(4):767-779 [FREE Full text] [doi: 10.1007/s11136-015-1122-4] [Medline: 26346986] 
29. Scherer RW, Saldanha IJ. How should systematic reviewers handle conference abstracts? A view from the trenches. Syst Rev 2019 Nov 7;8(1):264 [FREE Full text] [doi: 10.1186/s13643-019-1188-0] [Medline: 31699124]

30. Richards D. Handsearching still a valuable element of the systematic review. Evid Based Dent 2008;9(3):85. [doi: 10.1038/sj.ebd.6400602] [Medline: 18927572]

31. Terwee CB, Mokkink LB, Knol DL, Ostelo RW, Bouter LM, de Vet HC. Rating the methodological quality in systematic reviews of studies on measurement properties: a scoring system for the COSMIN checklist. Qual Life Res 2012 May;21(4):651-657 [FREE Full text] [doi: 10.1007/s11136-011-9960-1] [Medline: 21732199]

32. Mokkink L, Prinsen C, Patrick D. COSMIN methodology for systematic reviews of patient-reported outcome measures (PROMs). User manual 2018;78:1.

33. Vitolins MZ, Rand CS, Rapp SR, Ribisl PM, Sevick MA. Measuring adherence to behavioral and medical interventions. Control Clin Trials 2000 Oct;21(5 Suppl):188S-194S. [doi: 10.1016/s0197-2456(00)00077-5] [Medline: 11018574]

34. Smith H, Hankins M, Hodson A, George C. Measuring the adherence to medication of elderly patients with heart failure: is there a gold standard? Int J Cardiol 2010 Nov 5;145(1):122-123. [doi: 10.1016/j.ijcard.2009.06.031] [Medline: 19615767]

35. Mokkink LB, Terwee CB, Knol DL, Stratford PW, Alonso J, Patrick DL, et al. The COSMIN checklist for evaluating the methodological quality of studies on measurement properties: a clarification of its content. BMC Med Res Methodol 2010 Mar 18;10:22 [FREE Full text] [doi: 10.1186/1471-2288-10-22] [Medline: 20298572]

36. Terwee CB, Bot SD, de BM, van DW, Knol DL, Dekker J, et al. Quality criteria were proposed for measurement properties of health status questionnaires. J Clin Epidemiol 2007 Jan;60(1):34-42. [doi: 10.1016/j.jclinepi.2006.03.012] [Medline: 17161752]

37. Dijkers M. -. Introducing GRADE: a systematic approach to rating evidence in systematic reviews and to guideline development 2020:a.

38. Fredericksen R, Yang F, Gibbons L, Edwards T, Brown S, Fitzsimmons E, et al. Development and content validation of measures assessing adherence barriers and behaviors for use in clinical care. Res Social Adm Pharm 2019

Sep;15(9):1168-1176. [doi: 10.1016/j.sapharm.2018.10.001] [Medline: 30327183]

39. Axelsson M, Ekerljung L, Lundbäck B, Lötvall J. Personality and unachieved treatment goals related to poor adherence to asthma medication in a newly developed adherence questionnaire - a population-based study. Multidiscip Respir Med 2016;11:42 [FREE Full text] [doi: 10.1186/s40248-016-0078-8] [Medline: 27980735]

40. Chaiyachati K, Hirschhorn LR, Tanser F, Newell M, Bärnighausen T. Validating five questions of antiretroviral nonadherence in a public-sector treatment program in rural South Africa. AIDS Patient Care STDS 2011 Mar;25(3):163-170 [FREE Full text] [doi: 10.1089/apc.2010.0257] [Medline: 21269131]

41. Da W, Li X, Qiao S, Zhou Y, Shen Z. Evaluation of self-report adherence measures and their associations with detectable viral load among people living with HIV (PLHIV) in China. PLoS One 2018;13(8):e0203032 [FREE Full text] [doi: 10.1371/journal.pone.0203032] [Medline: 30161177]

42. Demirtaş A, Akbayrak N. Development of an assessment scale for treatment compliance in type 2 Diabetes Mellitus in Turkish population: Psychometric evaluation. International Journal of Nursing Sciences 2017 Jul;4(3):244-251. [doi: 10.1016/j.ijnss.2017.06.002]

43. Godin G, Gagné C, Naccache H. Validation of a self-reported questionnaire assessing adherence to antiretroviral medication. AIDS Patient Care STDS 2003 Jul;17(7):325-332. [doi: 10.1089/108729103322231268] [Medline: 12952734]

44. Kennedy SB. Developing a self-administered tool to predict adherence to antiretroviral therapy: design, method, and objectives. AIDS Patient Care STDS 2000 Jun;14(6):309-316. [doi: 10.1089/10872910050046331] [Medline: 10897503]

45. Kerr T, Hogg RS, Yip B, Tyndall MW, Montaner J, Wood E. Validity of self-reported adherence among injection drug users. J Int Assoc Physicians AIDS Care (Chic) 2008;7(4):157-159. [doi: 10.1177/1545109708320686] [Medline: 18626123]

46. Sidorkiewicz S, Tran V, Cousyn C, Perrodeau E, Ravaud P. Development and validation of an instrument to assess treatment adherence for each individual drug taken by a patient. BMJ Open 2016 May 10;6(5):e010510 [FREE Full text] [doi: 10.1136/bmjopen-2015-010510] [Medline: 27165645]

47. Tan J, Luo L, Zhang M, Chen H, Zhang D, Dong C, et al. A Chinese and Western medication adherence scale in patients with chronic kidney disease. Patient Prefer Adherence 2019;13:1487-1495 [FREE Full text] [doi: 10.2147/PPA.S207693] [Medline: $\underline{31507316}$ ]

48. Turcu-Stiolică A. Developing of a new tool for evaluation of therapeutic adherence in COPD. Farmacia 2018 Oct 13;66(5):920-924. [doi: 10.31925/farmacia.2018.5.25]

49. Cornelius T, Voils CI, Umland RC, Kronish IM. Validity of the self-reported domains of subjective extent of nonadherence (DOSE-nonadherence) scale in comparison with electronically monitored adherence to cardiovascular medications. Patient Prefer Adherence 2019;13:1677-1684 [FREE Full text] [doi: 10.2147/PPA.S225460] [Medline: 31631982]

50. Vreeman RC, Nyandiko WM, Ayaya SO, Walumbe EG, Inui TS. Cognitive interviewing for cross-cultural adaptation of pediatric antiretroviral therapy adherence measurement items. Int J Behav Med 2014 Feb;21(1):186-196. [doi: 10.1007/s12529-012-9283-9] [Medline: 23188670]

51. Wilson IB, Fowler FJ, Cosenza CA, Michaud J, Bentkover J, Rana A, et al. Cognitive and field testing of a new set of medication adherence self-report items for HIV care. AIDS Behav 2014 Dec;18(12):2349-2358 [FREE Full text] [doi: 10.1007/s10461-013-0610-1] [Medline: 24077970] 
52. Feldman BJ, Fredericksen RJ, Crane PK, Safren SA, Mugavero MJ, Willig JH, et al. Evaluation of the single-item self-rating adherence scale for use in routine clinical care of people living with HIV. AIDS Behav 2013 Jan;17(1):307-318 [FREE Full text] [doi: 10.1007/s10461-012-0326-7] [Medline: 23108721]

53. Wohl DA, Panter AT, Kirby C, Magnus BE, Hudgens MG, Allmon AG, et al. Estimating HIV medication adherence and persistence: two instruments for clinical and research use. AIDS Behav 2018 Mar;22(3):948-960. [doi:

10.1007/s10461-017-1772-z] [Medline: 28447269]

54. Unni EJ, Farris KB. Development of a new scale to measure self-reported medication nonadherence. Res Social Adm Pharm 2015;11(3):e133-e143. [doi: 10.1016/j.sapharm.2009.06.005] [Medline: 21272524]

55. Unni E, Sternbach N, Goren A. Using the medication adherence reasons scale (MAR-Scale) to identify the reasons for non-adherence across multiple disease conditions. Patient Prefer Adherence 2019;13:993-1004 [FREE Full text] [doi: 10.2147/PPA.S205359] [Medline: 31308635]

56. Kleppe M, Lacroix J, Ham J, Midden C. The development of the ProMAS: a probabilistic medication adherence scale. Patient Prefer Adherence 2015;9:355-367 [FREE Full text] [doi: 10.2147/PPA.S76749] [Medline: 25784791]

57. Dima AL, van Ganse E, Laforest L, Texier N, de Bruin M, The Astro-Lab Group. Measuring medication adherence in asthma: development of a novel self-report tool. Psychol Health 2017 Oct;32(10):1288-1307. [doi: 10.1080/08870446.2017.1290248] [Medline: 28276742]

58. Tran V, Harrington M, Montori VM, Barnes C, Wicks P, Ravaud P. Adaptation and validation of the treatment burden questionnaire (TBQ) in English using an internet platform. BMC Med 2014 Jul 2;12:109 [FREE Full text] [doi: 10.1186/1741-7015-12-109] [Medline: 24989988]

59. Wicks P, Massagli M, Kulkarni A, Dastani H. Use of an online community to develop patient-reported outcome instruments: the multiple sclerosis treatment adherence questionnaire (MS-TAQ). J Med Internet Res 2011 Jan 24;13(1):e12 [FREE Full text] [doi: 10.2196/jmir.1687] [Medline: 21266318]

60. Vale FC, Santa-Helena ET, Santos MA, Carvalho WM, Menezes PR, Basso CR, et al. Development and validation of the WebAd-questionnaire to monitor adherence to HIV therapy. Rev Saude Publica 2018;52:62 [FREE Full text] [doi: 10.11606/s1518-8787.2018052000337] [Medline: 29846437]

61. Schatz M, Zeiger RS, Yang S, Weinstein AG, Chen W, Saris-Baglama RN, et al. Development and preliminary validation of the adult asthma adherence questionnaire. J Allergy Clin Immunol Pract 2013;1(3):280-288. [doi: 10.1016/j.jaip.2013.03.001] [Medline: 24565486]

62. Buscher A, Hartman C, Kallen MA, Giordano TP. Validity of self-report measures in assessing antiretroviral adherence of newly diagnosed, HAART-naïve, HIV patients. HIV Clin Trials 2011;12(5):244-254 [FREE Full text] [doi: 10.1310/hct1205-244] [Medline: 22180522]

63. Gonzalez JS, Schneider HE, Wexler DJ, Psaros C, Delahanty LM, Cagliero E, et al. Validity of medication adherence self-reports in adults with type 2 diabetes. Diabetes Care 2013 Apr;36(4):831-837 [FREE Full text] [doi: 10.2337/dc12-0410] [Medline: 23204245]

64. Kalichman SC, Amaral CM, Swetzes C, Jones M, Macy R, Kalichman MO, et al. A simple single-item rating scale to measure medication adherence: further evidence for convergent validity. J Int Assoc Physicians AIDS Care (Chic) 2009;8(6):367-374 [FREE Full text] [doi: 10.1177/1545109709352884] [Medline: 19952289]

65. Reynolds NR, Sun J, Nagaraja HN, Gifford AL, Wu AW, Chesney MA. Optimizing measurement of self-reported adherence with the ACTG adherence questionnaire: a cross-protocol analysis. J Acquir Immune Defic Syndr 2007 Dec 1;46(4):402-409. [doi: 10.1097/qai.0b013e318158a44f] [Medline: 18077832]

66. Simoni JM, Huh D, Wang Y, Wilson IB, Reynolds NR, Remien RH, et al. The validity of self-reported medication adherence as an outcome in clinical trials of adherence-promotion interventions: findings from the MACH14 study. AIDS Behav 2014 Dec;18(12):2285-2290 [FREE Full text] [doi: 10.1007/s10461-014-0905-x] [Medline: 25280447]

67. Smith SR, Wahed AS, Kelley SS, Conjeevaram HS, Robuck PR, Fried MW, Virahep-C Study Group. Assessing the validity of self-reported medication adherence in hepatitis C treatment. Ann Pharmacother 2007 Jul;41(7):1116-1123. [doi: 10.1345/aph.1K024] [Medline: 17519299]

68. van den Boogaard J, Lyimo R, Boeree M, Kibiki G, Aarnoutse R. Electronic monitoring of treatment adherence and validation of alternative adherence measures in tuberculosis patients: a pilot study. Bull World Health Organ 2011 Sep 1;89(9):632-639 [FREE Full text] [doi: 10.2471/BLT.11.086462] [Medline: 21897483]

69. Chesney MA, Ickovics JR, Chambers DB, Gifford AL, Neidig J, Zwickl B, et al. Self-reported adherence to antiretroviral medications among participants in HIV clinical trials: the AACTG adherence instruments. Patient care committee \& adherence working group of the outcomes committee of the adult AIDS clinical trials group (AACTG). AIDS Care 2000 Jun;12(3):255-266. [doi: 10.1080/09540120050042891] [Medline: 10928201]

70. Gabriel A, Violato C. Knowledge of and attitudes towards depression and adherence to treatment: the antidepressant adherence scale (AAS). J Affect Disord 2010 Nov;126(3):388-394. [doi: 10.1016/j.jad.2010.07.013] [Medline: 20708273]

71. Müller S, Kohlmann T, Wilke T. Validation of the adherence barriers questionnaire - an instrument for identifying potential risk factors associated with medication-related non-adherence. BMC Health Serv Res 2015 Apr 10;15:153 [FREE Full text] [doi: 10.1186/s12913-015-0809-0] [Medline: 25884193] 
72. Mueller S, Wilke T, Gorasso V, Erhart M, Kittner JM. Adaption and validation of the adherence barriers questionnaire for HIV patients on antiretroviral therapy (ABQ-HIV). BMC Infect Dis 2018 Nov 28;18(1):599 [FREE Full text] [doi:

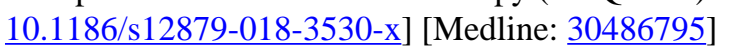

73. Daouphars M, Ouvry M, Lenain P, Rouvet J, Jardin F, Bubenheim M, et al. Preliminary validation of self-assessment tool to measure imatinib adherence in patients with chronic myeloid leukemia. Pharmacotherapy 2013 Feb;33(2):152-156. [doi: 10.1002/phar.1174] [Medline: 23359430]

74. Breuil V, Cortet B, Cotté FE, Arnould B, Dias-Barbosa C, Gaudin A, et al. Validation of the adherence evaluation of osteoporosis treatment (ADEOS) questionnaire for osteoporotic post-menopausal women. Osteoporos Int 2012 Feb;23(2):445-455. [doi: 10.1007/s00198-011-1555-8] [Medline: 21625889]

75. Martins MJ, Pinto AM, Castilho P, Macedo AF, Pereira AT, Bajouco M, et al. Assessing beliefs and attitudes towards antipsychotic medication from a recovery-based perspective: psychometric properties of a new scale. Psychiatry Res 2019 Mar;273:325-330. [doi: 10.1016/j.psychres.2019.01.043] [Medline: 30677722]

76. Ayiesah R, Leonard J, Chong C. Development and validation of non-adherence to pulmonary rehabilitation questionnaire: a clinical tool for patients with chronic obstructive pulmonary diseases. Clin Ter 2014;165(3):123-128. [doi: 10.7417/CT.2014.1708] [Medline: 24999563]

77. Kripalani S, Risser J, Gatti ME, Jacobson TA. Development and evaluation of the adherence to refills and medications scale (ARMS) among low-literacy patients with chronic disease. Value Health 2009;12(1):118-123 [FREE Full text] [doi: 10.1111/j.1524-4733.2008.00400.x] [Medline: 19911444$]$

78. Gökdoğan F, Kes D. Validity and reliability of the Turkish adherence to refills and medications scale. Int J Nurs Pract 2017 Oct;23(5). [doi: 10.1111/ijn.12566] [Medline: 28726281]

79. Matza LS, Park J, Coyne KS, Skinner EP, Malley KG, Wolever RQ. Derivation and validation of the ASK-12 adherence barrier survey. Ann Pharmacother 2009 Oct;43(10):1621-1630. [doi: 10.1345/aph.1M174] [Medline: 19776298]

80. Atsuta R, To Y, Sakamoto S, Mukai I, Kobayashi A, Kinoshita A, et al. Assessing usability of the 'adherence starts with knowledge 20' (ASK-20) questionnaire for Japanese adults with bronchial asthma receiving inhaled corticosteroids long term. Allergol Int 2017 Jul;66(3):411-417 [FREE Full text] [doi: 10.1016/j.alit.2016.09.001] [Medline: 27712949]

81. Hahn SR, Park J, Skinner EP, Yu-Isenberg KS, Weaver MB, Crawford B, et al. Development of the ASK-20 adherence barrier survey. Curr Med Res Opin 2008 Jul;24(7):2127-2138. [doi: 10.1185/03007990802174769] [Medline: 18554431]

82. Matza LS, Yu-Isenberg KS, Coyne KS, Park J, Wakefield J, Skinner EP, et al. Further testing of the reliability and validity of the ASK-20 adherence barrier questionnaire in a medical center outpatient population. Curr Med Res Opin 2008 Nov;24(11):3197-3206. [doi: 10.1185/03007990802463642] [Medline: 18922213]

83. Zeller A, Schroeder K, Peters TJ. An adherence self-report questionnaire facilitated the differentiation between nonadherence and nonresponse to antihypertensive treatment. J Clin Epidemiol 2008 Mar;61(3):282-288. [doi: 10.1016/j.jclinepi.2007.04.007] [Medline: 18226752]

84. Moss AC, Lillis Y, Edwards George JB, Choudhry NK, Berg AH, Cheifetz AS, et al. Attitudes to mesalamine questionnaire: a novel tool to predict mesalamine nonadherence in patients with IBD. Am J Gastroenterol 2014 Dec;109(12):1850-1855. [doi: 10.1038/ajg.2014.158] [Medline: 24913040]

85. Marsicano ED, Fernandes ND, Colugnati F, Grincenkov FR, Fernandes NM, De Geest S, et al. Transcultural adaptation and initial validation of Brazilian-Portuguese version of the Basel assessment of adherence to immunosuppressive medications scale (BAASIS) in kidney transplants. BMC Nephrol 2013 May 21;14:108 [FREE Full text] [doi: 10.1186/1471-2369-14-108] [Medline: 23692889]

86. Dolder CR, Lacro JP, Warren KA, Golshan S, Perkins DO, Jeste DV. Brief evaluation of medication influences and beliefs: development and testing of a brief scale for medication adherence. J Clin Psychopharmacol 2004 Aug;24(4):404-409. [doi: 10.1097/01.jcp.0000130554.63254.3a] [Medline: 15232332]

87. McDonald-Miszczak L, Maris P, Fitzgibbon T, Ritchie G. A pilot study examining older adults' beliefs related to medication adherence: the BERMA survey. J Aging Health 2004 Nov;16(5):591-614. [doi: 10.1177/0898264304265772] [Medline: 15448274]

88. Chui E, Wong K, Chan K, Wong M. Validation study of the brief medication adherence scale (BMAS) in patients with schizophrenia and related disorders in Hong Kong. Asian J Psychiatr 2018 Oct;37:154-160. [doi: 10.1016/j.ajp.2018.09.005] [Medline: 30265964]

89. Bennett SJ, Milgrom LB, Champion V, Huster GA. Beliefs about medication and dietary compliance in people with heart failure: an instrument development study. Heart Lung 1997;26(4):273-279. [doi: 10.1016/s0147-9563(97)90084-4] [Medline: 9257137]

90. Sriwarakorn S, Krittiyanunt S, Sakulbumrungsil R. SENSITIVITY AND SPECIFICITY OF THAI-VERSION BRIEF MEDICATION QUESTIONNAIRE. Journal of Health Research. Jul-Sep 2010;24(3):129-134.

91. Svarstad BL, Chewning BA, Sleath BL, Claesson C. The brief medication questionnaire: a tool for screening patient adherence and barriers to adherence. Patient Educ Couns 1999 Jun;37(2):113-124. [doi: 10.1016/s0738-3991(98)00107-4] [Medline: 14528539] 
92. Choo PW, Rand CS, Inui TS, Lee MT, Cain E, Cordeiro-Breault M, et al. Validation of patient reports, automated pharmacy records, and pill counts with electronic monitoring of adherence to antihypertensive therapy. Med Care 1999 Sep;37(9):846-857. [doi: 10.1097/00005650-199909000-00002] [Medline: 10493464]

93. Kerr SJ, Avihingsanon A, Putcharoen O, Chetchotisakd P, Layton M, Ubolyam S, et al. Assessing adherence in Thai patients taking combination antiretroviral therapy. Int J STD AIDS 2012 Mar;23(3):160-165. [doi: 10.1258/ijsa.2009.009152] [Medline: 22581867]

94. Mannheimer SB, Mukherjee R, Hirschhorn LR, Dougherty J, Celano SA, Ciccarone D, et al. The CASE adherence index: a novel method for measuring adherence to antiretroviral therapy. AIDS Care 2006 Oct;18(7):853-861 [FREE Full text] [doi: 10.1080/09540120500465160] [Medline: 16971298 ]

95. Kyngäs HA, Skaar-Chandler CA, Duffy ME. The development of an instrument to measure the compliance of adolescents with a chronic disease. J Adv Nurs 2000 Dec;32(6):1499-1506. [doi: 10.1046/j.1365-2648.2000.01611.x] [Medline:

$\underline{11136419]}$

96. Wales S, Crisp J, Fernandes R, Kyngas H. Modification and testing of the chronic disease compliance instrument to measure treatment compliance in adolescents with asthma. Contemp Nurse 2011 Oct;39(2):147-156. [doi: 10.5172/conu.2011.147] [Medline: 22551427]

97. Fernandes R, Wales S, Crisp J, Kyngas H. Modification and testing of the chronic disease compliance instrument to measure treatment compliance in adolescents with diabetes. J Clin Nurs 2011 May;20(9-10):1273-1281. [doi:

10.1111/j.1365-2702.2010.03566.x] [Medline: 21492273]

98. Dima AL, Schweitzer A, Diaconiţ R, Remor E, Wanless RS. Adherence to ARV medication in Romanian young adults: self-reported behaviour and psychological barriers. Psychol Health Med 2013;18(3):343-354. [doi: 10.1080/13548506.2012.722648] [Medline: 22985131]

99. Kampman O, Lehtinen K, Lassila V. The reliability of compliance assessments performed by doctors and patients during neuroleptic treatment: a comparison of compliance ratings. Acta Psychiatr Scand Oct 2001;104(4):-. [doi: 10.1111/j.1600-0447.2001.00159.x]

100. Krousel-Wood M, Joyce C, Holt EW, Levitan EB, Dornelles A, Webber LS, et al. Development and evaluation of a self-report tool to predict low pharmacy refill adherence in elderly patients with uncontrolled hypertension. Pharmacotherapy 2013 Aug;33(8):798-811 [FREE Full text] [doi: 10.1002/phar.1275] [Medline: 23649849]

101. de Klerk E, van der Heijde D, van der Tempel H, van der Linden S. Development of a questionnaire to investigate patient compliance with antirheumatic drug therapy. J Rheumatol 1999 Dec;26(12):2635-2641. [Medline: 10606375]

102. Salt E, Hall L, Peden AR, Home R. Psychometric properties of three medication adherence scales in patients with rheumatoid arthritis. J Nurs Meas 2012;20(1):59-72. [doi: 10.1891/1061-3749.20.1.59] [Medline: 22679710]

103. Lee JY, Lee SY, Hahn HJ, Son IJ, Hahn SG, Lee EB. Cultural adaptation of a compliance questionnaire for patients with rheumatoid arthritis to a Korean version. Korean J Intern Med 2011 Mar;26(1):28-33 [FREE Full text] [doi: 10.3904/kjim.2011.26.1.28] [Medline: 21437159]

104. Cinar F, Cinar M, Yilmaz S, Acikel C, Erdem H, Pay S, et al. AB0658 Cross-cultural adaptation, reliability, and validity of the turkish version of the compliance questionnaire on rheumatology (CQR-T) in patients with Behçet's disease. Ann Rheum Dis 2015 Jun 9;74(Suppl 2):1118.3-1118.9. [doi: 10.1136/annrheumdis-2015-eular.3171]

105. Hughes LD, Done J, Young A. A 5 item version of the compliance questionnaire for rheumatology (CQR5) successfully identifies low adherence to DMARDs. BMC Musculoskelet Disord 2013 Oct 8;14:286 [FREE Full text] [doi: 10.1186/1471-2474-14-286] [Medline: 24103582]

106. Hogan TP, Awad AG, Eastwood R. A self-report scale predictive of drug compliance in schizophrenics: reliability and discriminative validity. Psychol Med 1983 Feb;13(1):177-183. [doi: 10.1017/s0033291700050182] [Medline: 6133297]

107. Friemann K, Wciórka J. [Four measures of treatment compliance among patients recovering from psychotic episodes--a comparative study]. Psychiatr Pol 2013;47(5):759-773. [doi: 10.12740/pp/18341] [Medline: 25011225]

108. Kikkert MJ, Koeter MW, Dekker JJ, Burti L, Robson D, Puschner B, et al. The predictive validity of subjective adherence measures in patients with schizophrenia. Int J Methods Psychiatr Res 2011 Jun;20(2):73-81 [FREE Full text] [doi: 10.1002/mpr.335] [Medline: 21557378]

109. Stjernswärd S, Persson K, Nielsen R, Tuninger E, Levander S. A modified drug attitude inventory used in long-term patients in sheltered housing. Eur Neuropsychopharmacol 2013 Oct;23(10):1296-1299. [doi: 10.1016/j.euroneuro.2012.11.011] [Medline: 23265955]

110. Garfield S, Eliasson L, Clifford S, Willson A, Barber N. Developing the diagnostic adherence to medication scale (the DAMS) for use in clinical practice. BMC Health Serv Res 2012 Oct 8;12:350 [FREE Full text] [doi: 10.1186/1472-6963-12-350] [Medline: 23039138]

111. Jacobsen R, Møldrup C, Christrup L, Sjøgren P, Hansen O. The Danish version of the medication adherence report scale: preliminary validation in cancer pain patients. Pain Pract 2009;9(1):1-7. [doi: 10.1111/j.1533-2500.2008.00245.x] [Medline: $\underline{19019056]}$

112. Mehta SN, Nansel TR, Volkening LK, Butler DA, Haynie DL, Laffel LM. Validation of a contemporary adherence measure for children with type 1 diabetes: the diabetes management questionnaire. Diabet Med 2015 Sep;32(9):1232-1238 [FREE Full text] [doi: 10.1111/dme.12682] [Medline: 26280463] 
113. Sleath B, Carpenter D, Blalock S, Davis S, Hickson R, Lee C, et al. Development of a new diabetes medication self-efficacy scale and its association with both reported problems in using diabetes medications and self-reported adherence. Patient Prefer Adherence 2016;10:1003-1010 [FREE Full text] [doi: 10.2147/PPA.S101349] [Medline: 27354769]

114. Zheng J, Wang Y, Ye X, Xiao L, Ye J, Li X, et al. Validation of diabetes medication self-efficacy scale in Chinese with type 2 diabetes. Patient Prefer Adherence 2018;12:2517-2525 [FREE Full text] [doi: 10.2147/PPA.S170144] [Medline: 30568430]

115. Irvine AA, Saunders JT, Blank MB, Carter WR. Validation of scale measuring environmental barriers to diabetes-regimen adherence. Diabetes Care 1990 Jul;13(7):705-711. [doi: 10.2337/diacare.13.7.705] [Medline: 2387191]

116. Regnault A, Viala-Danten M, Gilet H, Berdeaux G. Scoring and psychometric properties of the eye-drop satisfaction questionnaire (EDSQ), an instrument to assess satisfaction and compliance with glaucoma treatment. BMC Ophthalmol 2010 Feb 1;10:1 [FREE Full text] [doi: 10.1186/1471-2415-10-1] [Medline: 20122146]

117. Nordmann J, Denis P, Vigneux M, Trudeau E, Guillemin I, Berdeaux G. Development of the conceptual framework for the eye-drop satisfaction questionnaire (EDSQ) in glaucoma using a qualitative study. BMC Health Serv Res 2007 Aug 6;7:124 [FREE Full text] [doi: 10.1186/1472-6963-7-124] [Medline: 17683594]

118. Kim Y, Evangelista LS, Phillips LR, Pavlish C, Kopple JD. The end-stage renal disease adherence questionnaire (ESRD-AQ): testing the psychometric properties in patients receiving in-center hemodialysis. Nephrol Nurs J 2010;37(4):377-393 [FREE Full text] [Medline: 20830945]

119. Poveda V, Amado L, Filgueiras M, Teixeira L, Miranda V, Santos-Silva A, et al. End-stage renal disease adherence questionnaire: translation and validation to the Portuguese language. Ren Fail 2016 Nov;38(10):1633-1638. [doi: 10.1080/0886022X.2016.1209063] [Medline: 27764984]

120. Fairley C, Permana A, Read T. Long-term utility of measuring adherence by self-report compared with pharmacy record in a routine clinic setting. HIV Med 2005 Sep;6(5):366-369 [FREE Full text] [doi: 10.1111/j.1468-1293.2005.00322.x] [Medline: 16156886]

121. Kandrotaite K, Smigelskas K, Janusauskiene D, Jievaltas M, Maciulaitis R, Briedis V. Development of a short questionnaire to identify the risk of nonadherence to antibiotic treatment. Curr Med Res Opin 2013 Nov;29(11):1555-1563. [doi: 10.1185/03007995.2013.835255] [Medline: 23952367]

122. Zongo A, Guénette L, Moisan J, Grégoire JP. Predictive validity of self-reported measures of adherence to noninsulin antidiabetes medication against control of glycated hemoglobin levels. Can J Diabetes 2016 Feb;40(1):58-65. [doi: 10.1016/j.jcjd.2015.06.008] [Medline: 26507401]

123. Naqvi AA, AlShayban DM, Ghori SA, Mahmoud MA, Haseeb A, Faidah HS, et al. Validation of the general medication adherence scale in Saudi patients with chronic diseases. Front Pharmacol 2019;10:633 [FREE Full text] [doi: 10.3389/fphar.2019.00633] [Medline: 31231222]

124. Naqvi AA, Hassali MA, Jahangir A, Nadir MN, Kachela B. Translation and validation of the English version of the general medication adherence scale (GMAS) in patients with chronic illnesses. J Drug Assess 2019;8(1):36-42 [FREE Full text] [doi: $\underline{10.1080 / 21556660.2019 .1579729]}$ [Medline: $\underline{\text { 30863660] }}$

125. Naqvi AA, Hassali MA, Rizvi M, Zehra A, Iffat W, Haseeb A, et al. Development and validation of a novel general medication adherence scale (GMAS) for chronic illness patients in Pakistan. Front Pharmacol 2018 Oct 9;9:1124 [FREE Full text] [doi: 10.3389/fphar.2018.01124] [Medline: $\underline{\text { 30356775] }}$

126. Mansberger SL, Sheppler CR, McClure TM, Vanalstine CL, Swanson IL, Stoumbos Z, et al. Psychometrics of a new questionnaire to assess glaucoma adherence: the glaucoma treatment compliance assessment tool (an American ophthalmological society thesis). Trans Am Ophthalmol Soc 2013 Sep;111:1-16 [FREE Full text] [Medline: 24072942]

127. Abe RY, Wen LD, Barker GT, Mansberger SL. Psychometric Properties of the glaucoma treatment compliance assessment tool (GTCAT) in a Brazilian population. J Glaucoma 2018 Mar;27(3):257-265. [doi: 10.1097/IJG.0000000000000876] [Medline: 29369851]

128. Barker GT, Mansberger SL. Psychometric properties of the reduced version of the glaucoma treatment compliance assessment tool (GTCAT). Ophthalmic Epidemiol 2019 Feb;26(1):55-62. [doi: 10.1080/09286586.2018.1516785] [Medline: 30204034]

129. Song Y, Han H, Song H, Nam S, Nguyen T, Kim MT. Psychometric evaluation of hill-bone medication adherence subscale. Asian Nurs Res (Korean Soc Nurs Sci) 2011 Sep;5(3):183-188 [FREE Full text] [doi: 10.1016/j.anr.2011.09.007] [Medline: 25030368]

130. Koschack J, Marx G, Schnakenberg J, Kochen MM, Himmel W. Comparison of two self-rating instruments for medication adherence assessment in hypertension revealed insufficient psychometric properties. J Clin Epidemiol 2010 Mar;63(3):299-306. [doi: 10.1016/j.jclinepi.2009.06.011] [Medline: 19762213]

131. Krousel-Wood M, Muntner P, Jannu A, Desalvo K, Re RN. Reliability of a medication adherence measure in an outpatient setting. Am J Med Sci 2005 Sep;330(3):128-133. [doi: 10.1097/00000441-200509000-00006] [Medline: 16174996]

132. Nogueira-Silva L, Sá-Sousa A, Lima MJ, Monteiro A, Dennison-Himmelfarb C, Fonseca JA. Translation and cultural adaptation of the hill-bone compliance to high blood pressure therapy scale to Portuguese. Rev Port Cardiol 2016 Feb;35(2):93-97 [FREE Full text] [doi: 10.1016/j.repc.2015.07.013] [Medline: 26852304]

133. Alsolami FJ, Hou X, Correa-Velez I. An arabic instrument to measure medication adherence in Saudi hypertensive patients. ME-JFM 2013 Sep;11(7):17-23. [doi: 10.5742/mefm.2014.92409] 
134. Nelsen A, Trautner BW, Petersen NJ, Gupta S, Rodriguez-Barradas M, Giordano TP, et al. Development and validation of a measure for intention to adhere to HIV treatment. AIDS Patient Care STDS 2012 Jun;26(6):329-334 [FREE Full text] [doi: 10.1089/apc.2011.0318] [Medline: 22680281]

135. Spire B, Arnould B, Barbier F, Durant J, Gilquin J, Landman R, et al. Simplification and first validation of a short battery of patient questionnaires for clinical management of HIV-infected patients: the HIV-SQUAD (symptom quality of life adherence) questionnaire. HIV Clin Trials 2009;10(4):215-232. [doi: 10.1310/hct1004-215] [Medline: 19723610]

136. Mikhael EM, Hussain SA, Shawky N, Hassali MA. Validity and reliability of anti-diabetic medication adherence scale among patients with diabetes in Baghdad, Iraq: a pilot study. BMJ Open Diabetes Res Care 2019;7(1):e000658 [FREE Full text] [doi: 10.1136/bmjdrc-2019-000658] [Medline: 31354953]

137. Jesus-Nunes AP, Morais-de-Jesus M, Dantas-Duarte A, Moreira TM, Argolo FC, Castro AD, et al. The Portuguese version of the immunosuppressant therapy adherence scale (ITAS) among liver transplant recipient patients: translation and psychometric properties. Ann Hepatol 2018;17(1):104-109 [FREE Full text] [doi: 10.5604/01.3001.0010.7541] [Medline: 29311393]

138. Wilks S, Spivey C, Chisholm-Burns M. Psychometric re-evaluation of the immunosuppressant therapy adherence scale among solid-organ transplant recipients. J Eval Clin Pract 2010 Feb;16(1):64-68. [doi: 10.1111/j.1365-2753.2008.01115.x] [Medline: 20367816]

139. Chisholm MA, Lance CE, Williamson GM, Mulloy LL. Development and validation of an immunosuppressant therapy adherence barrier instrument. Nephrol Dial Transplant 2005 Jan;20(1):181-188. [doi: 10.1093/ndt/gfh576] [Medline: 15572384]

140. de Geest S, Abraham I, Gemoets H, Evers G. Development of the long-term medication behaviour self-efficacy scale: qualitative study for item development. J Adv Nurs 1994 Feb;19(2):233-238. [doi: 10.1111/j.1365-2648.1994.tb01076.x] [Medline: 8188953]

141. Lee S, Bae Y, Worley M, Law A. Validating the modified drug adherence work-up (M-DRAW) tool to identify and address barriers to medication adherence. Pharmacy (Basel) 2017 Sep 8;5(3):- [FREE Full text] [doi: 10.3390/pharmacy5030052] [Medline: 28970464]

142. Lee S, Bae-Shaaw YH, Gogineni H, Worley MM, Law AV. Triple strength utility of the modified drug adherence work-up (M-DRAW) tool in a veterans affairs outpatient diabetes clinic. Res Social Adm Pharm 2020 Jul;16(7):914-920. [doi: 10.1016/j.sapharm.2019.09.063] [Medline: 31629655]

143. Sadakathulla I, Mateti U, Kellarai A, Bhat K. Adhering to antihypertensive treatment is vitally important. Article Salud(i)Ciencia 2019;23(4):314-324. [doi: 10.21840/siic/157368]

144. Unni EJ, Olson JL, Farris KB. Revision and validation of medication adherence reasons scale (MAR-scale). Curr Med Res Opin 2014 Feb;30(2):211-221. [doi: 10.1185/03007995.2013.851075] [Medline: 24102296]

145. Unni E, Sternbach N, Goren A. Using the medication adherence reasons scale (MAR-Scale) to identify the reasons for non-adherence across multiple disease conditions. Patient Prefer Adherence 2019;13:993-1004 [FREE Full text] [doi: 10.2147/PPA.S205359] [Medline: 31308635]

146. Fond G, Boyer L, Boucekine M, Aden LA, Schürhoff F, Tessier A, FACE-SZ (Fonda and Mental Academic Centers of Expertise for Schizophrenia) group. Validation study of the medication adherence rating scale. Results from the FACE-SZ national dataset. Schizophr Res 2017 Apr;182:84-89. [doi: 10.1016/j.schres.2016.10.023] [Medline: 27789187]

147. Kao Y, Liu Y. Compliance and schizophrenia: the predictive potential of insight into illness, symptoms, and side effects. Compr Psychiatry 2010;51(6):557-565. [doi: 10.1016/j.comppsych.2010.03.007] [Medline: 20965300]

148. Owie GO, Olotu SO, James BO. Reliability and validity of the medication adherence rating scale in a cohort of patients with schizophrenia from Nigeria. Trends Psychiatry Psychother 2018;40(2):85-92 [FREE Full text] [doi: 10.1590/2237-6089-2017-0077] [Medline: 29768528]

149. Sowunmi O, Onifade P. Psychometric evaluation of medication adherence rating scale (MARS) among Nigerian patients with schizophrenia. Niger J Clin Pract 2019 Sep;22(9):1281-1285 [FREE Full text] [doi: 10.4103/njcp.njcp 325 18] [Medline: 31489867$]$

150. Zemmour K, Tinland A, Boucekine M, Girard V, Loubière S, Resseguier N, French Housing First Study Group. Validation of the medication adherence rating scale in homeless patients with schizophrenia: results from the French housing first experience. Sci Rep 2016 Aug 18;6:31598 [FREE Full text] [doi: 10.1038/srep31598] [Medline: 27534796]

151. Cohen JL, Mann DM, Wisnivesky JP, Home R, Leventhal H, Musumeci-Szabó TJ, et al. Assessing the validity of self-reported medication adherence among inner-city asthmatic adults: the medication adherence report scale for asthma. Ann Allergy Asthma Immunol 2009 Oct;103(4):325-331. [doi: 10.1016/s1081-1206(10)60532-7] [Medline: 19852197]

152. Alsous M, Alhalaiqa F, Abu Farha R, Abdel Jalil M, McElnay J, Horne R. Reliability and validity of arabic translation of medication adherence report scale (MARS) and beliefs about medication questionnaire (BMQ)-specific for use in children and their parents. PLoS One 2017;12(2):e0171863 [FREE Full text] [doi: 10.1371/journal.pone.0171863] [Medline: 28192467]

153. Garcia-Marcos PW, Brand PL, Kaptein AA, Klok T. Is the MARS questionnaire a reliable measure of medication adherence in childhood asthma? J Asthma 2016 Dec;53(10):1085-1089. [doi: 10.1080/02770903.2016.1180699] [Medline: 27177241] 
154. Jónsdóttir H, Opjordsmoen S, Birkenaes AB, Engh JA, Ringen PA, Vaskinn A, et al. Medication adherence in outpatients with severe mental disorders: relation between self-reports and serum level. J Clin Psychopharmacol 2010 Apr;30(2):169-175. [doi: 10.1097/JCP.0b013e3181d2191e] [Medline: 20520290]

155. Lee L, El-Den S, Horne R, Carter SR. Patient satisfaction with information, concerns, beliefs and adherence to topical corticosteroids. Patient Educ Couns 2019 Jun;102(6):1203-1209. [doi: 10.1016/j.pec.2019.01.019] [Medline: 30928342]

156. Lin C, Ou H, Nikoobakht M, Broström A, Årestedt K, Pakpour A. Validation of the 5-item medication adherence report scale in older stroke patients in Iran. J Cardiovasc Nurs 2018;33(6):536-543. [doi: 10.1097/JCN.0000000000000488] [Medline: 29649015]

157. Tommelein E, Mehuys E, van Tongelen I, Brusselle G, Boussery K. Accuracy of the medication adherence report scale (MARS-5) as a quantitative measure of adherence to inhalation medication in patients with COPD. Ann Pharmacother 2014 May;48(5):589-595. [doi: 10.1177/1060028014522982] [Medline: 24523393]

158. van de Steeg N, Sielk M, Pentzek M, Bakx C, Altiner A. Drug-adherence questionnaires not valid for patients taking blood-pressure-lowering drugs in a primary health care setting. J Eval Clin Pract 2009 Jun;15(3):468-472. [doi: 10.1111/j.1365-2753.2008.01038.x] [Medline: 19366389]

159. Mahler C, Hermann K, Horne R, Ludt S, Haefeli WE, Szecsenyi J, et al. Assessing reported adherence to pharmacological treatment recommendations. Translation and evaluation of the medication adherence report scale (MARS) in Germany. J Eval Clin Pract 2010 Jun;16(3):574-579. [doi: 10.1111/j.1365-2753.2009.01169.x] [Medline: 20210821]

160. Sampaio R, Azevedo LF, Dias CC, Horne R, Castro Lopes JM. Portuguese version of the medication adherence report scale (MARS-9): validation in a population of chronic pain patients. J Eval Clin Pract 2019 Apr;25(2):346-352. [doi: 10.1111/jep.13098] [Medline: 30648328]

161. Ogedegbe G, Mancuso CA, Allegrante JP, Charlson ME. Development and evaluation of a medication adherence self-efficacy scale in hypertensive African-American patients. J Clin Epidemiol 2003 Jun;56(6):520-529. [doi: 10.1016/s0895-4356(03)00053-2] [Medline: 12873646]

162. Saffari M, Zeidi IM, Fridlund B, Chen H, Pakpour AH. A persian adaptation of medication adherence self-efficacy scale (MASES) in hypertensive patients: psychometric properties and factor structure. High Blood Press Cardiovasc Prev 2015 Sep;22(3):247-255. [doi: 10.1007/s40292-015-0101-8] [Medline: 25986076]

163. Andy UU, Harvie HS, Smith AL, Propert KJ, Bogner HR, Arya LA. Validation of a self-administered instrument to measure adherence to anticholinergic drugs in women with overactive bladder. Neurourol Urodyn 2015 Jun;34(5):424-428 [FREE Full text] [doi: 10.1002/nau.22605] [Medline: 24719232]

164. Koneru S, Shishov M, Ware A, Farhey Y, Mongey A, Graham TB, et al. Effectively measuring adherence to medications for systemic lupus erythematosus in a clinical setting. Arthritis Rheum 2007 Aug 15;57(6):1000-1006 [FREE Full text] [doi: 10.1002/art.22898] [Medline: 17665465$]$

165. Kosilov K, Loparev S, Kuzina I, Kosilova L, Ivanovskaya M, Prokofyeva A. Effectiveness of a new tool for self-evaluation of adherence to antimuscarinic drug treatment in older patients of both sexes with urge incontinence. Geriatr Gerontol Int 2018 Jan;18(1):115-122. [doi: 10.1111/ggi.13150] [Medline: 28921819]

166. Kosilov K, Loparev S, Kuzina I, Shakirova O, Zhuravskaya N, Lobodenko A. Self-assessment of treatment compliance with antimuscarinic drugs and lower urinary tract condition among women with urinary incontinence. Int Urogynecol J 2017 Nov;28(11):1663-1669. [doi: 10.1007/s00192-017-3333-4] [Medline: 28429053]

167. Kosilov K, Loparev S, Kuzina I, Shakirova O, Zhuravskaya N, Lobodenko A. The effective tool for self-assessment of adherence to treatment in patients with benign prostatic obstruction and overactive bladder symptoms. Aging Male 2017 Mar;20(1):39-44. [doi: 10.1080/13685538.2016.1247435] [Medline: 28074677]

168. Kosilov KV, Loparev SA, Kuzina IG, Shakirova OV, Gainullina YI, Kosilova LV, et al. A new tool for self-evaluation of adherence to antimuscarinic drugs treatment in patients with urinary incontinence. Arab J Urol 2017 Dec;15(4):372-379 [FREE Full text] [doi: 10.1016/j.aju.2017.10.007] [Medline: 29234543]

169. Ueno H, Yamazaki Y, Yonekura Y, Park M, Ishikawa H, Kiuchi T. Reliability and validity of a 12-item medication adherence scale for patients with chronic disease in Japan. BMC Health Serv Res 2018 Jul 31;18(1):592 [FREE Full text] [doi: 10.1186/s12913-018-3380-7] [Medline: 30064422]

170. Battistella M, Fleites R, Wong R, Jassal SV. Development, validation, and implementation of a medication adherence survey to seek a better understanding of the hemodialysis patient. Clin Nephrol 2016 Jan;85(1):12-22. [doi: 10.5414/CN108654] [Medline: 26636327]

171. Athavale AS, Bentley JP, Banahan BF, McCaffrey DJ, Pace PF, Vorhies DW. Development of the medication adherence estimation and differentiation scale (MEDS). Curr Med Res Opin 2019 Apr;35(4):577-585. [doi: 10.1080/03007995.2018.1512478] [Medline: $\underline{\text { 30106315] }}$

172. Cate H, Bhattacharya D, Clark A, Holland R, Broadway DC. A comparison of measures used to describe adherence to glaucoma medication in a randomised controlled trial. Clin Trials 2015 Dec;12(6):608-617. [doi: 10.1177/1740774515592636] [Medline: 26183437]

173. Cohen HW, Shmukler C, Ullman R, Rivera CM, Walker EA. Measurements of medication adherence in diabetic patients with poorly controlled $\mathrm{HbA}(1 \mathrm{c})$. Diabet Med $2010 \mathrm{Feb}$;27(2):210-216 [FREE Full text] [doi: 10.1111/j.1464-5491.2009.02898.x] [Medline: 20546266] 
174. Guénette L, Moisan J, Préville M, Boyer R. Measures of adherence based on self-report exhibited poor agreement with those based on pharmacy records. J Clin Epidemiol 2005 Sep;58(9):924-933. [doi: 10.1016/j.jclinepi.2005.02.002] [Medline: $\underline{16085196]}$

175. Morisky DE, Green LW, Levine DM. Concurrent and predictive validity of a self-reported measure of medication adherence. Med Care 1986 Jan;24(1):67-74. [doi: 10.1097/00005650-198601000-00007] [Medline: 3945130]

176. Shalansky SJ, Levy AR, Ignaszewski AP. Self-reported Morisky score for identifying nonadherence with cardiovascular medications. Ann Pharmacother 2004 Sep;38(9):1363-1368. [doi: 10.1345/aph.1E071] [Medline: 15238622]

177. Brooks CM, Richards JM, Kohler CL, Soong S, Martin B, Windsor RA, et al. Assessing adherence to asthma medication and inhaler regimens: a psychometric analysis of adult self-report scales. Med Care 1994 Mar;32(3):298-307. [doi: 10.1097/00005650-199403000-00008] [Medline: 8145604]

178. Erickson SR, Coombs JH, Kirking DM, Azimi AR. Compliance from self-reported versus pharmacy claims data with metered-dose inhalers. Ann Pharmacother 2001 Sep;35(9):997-1003. [doi: 10.1345/aph.10379] [Medline: 11573875]

179. Kristina SA, Putri LR, Riani DA, Ikawati Z, Endarti D. Validity of self-reported measure of medication adherence among diabetic patients in Indonesia. Int Res J Pharm 2019 Aug 2;10(7):144-148. [doi: 10.7897/2230-8407.1007234]

180. Wang Y, Lee J, Toh M, Tang W, Ko Y. Validity and reliability of a self-reported measure of medication adherence in patients with type 2 diabetes mellitus in Singapore. Diabet Med 2012 Sep;29(9):e338-e344. [doi: 10.1111/j.1464-5491.2012.03733.x] [Medline: 22672497]

181. Prado JC, Kupek E, Mion D. Validity of four indirect methods to measure adherence in primary care hypertensives. J Hum Hypertens 2007 Jul;21(7):579-584. [doi: 10.1038/sj.jhh.1002196] [Medline: 17443212]

182. Jerant A, DiMatteo R, Arnsten J, Moore-Hill M, Franks P. Self-report adherence measures in chronic illness: retest reliability and predictive validity. Med Care 2008 Nov;46(11):1134-1139. [doi: 10.1097/MLR.0b013e31817924e4] [Medline: 18953223]

183. Fahey M, Abdulmajeed A, Sabra K. Measurement of adherence to anti-hypertensive medication as perceived by doctors and patients. Qatar Med J 2006 Jun;2006(1):16. [doi: 10.5339/qmj.2006.1.16]

184. Ali MA, Abou-Taleb DA, Mohamed RR. Treatment adherence and beliefs about medicines among Egyptian vitiligo patients. Dermatol Ther 2016 Nov;29(6):413-418. [doi: 10.1111/dth.12397] [Medline: 27594551]

185. Pandey A, Raza F, Velasco A, Brinker S, Ayers C, Das SR, et al. Comparison of Morisky medication adherence scale with therapeutic drug monitoring in apparent treatment-resistant hypertension. J Am Soc Hypertens 2015 Jun;9(6):420-6.e2. [doi: 10.1016/j.jash.2015.04.004] [Medline: 26051923]

186. Ashur S, Shamsuddin K, Shah S, Bosseri S, Morisky DE. Reliability and known-group validity of the Arabic version of the 8-item Morisky medication adherence scale among type 2 diabetes mellitus patients. East Mediterr Health J 2015 Dec 13;21(10):722-728. [doi: 10.26719/2015.21.10.722] [Medline: 26750162]

187. de las Cuevas C, Peñate W. Psychometric properties of the eight-item Morisky medication adherence scale (MMAS-8) in a psychiatric outpatient setting. Int J Clin Health Psychol 2015;15(2):121-129 [FREE Full text] [doi: 10.1016/j.ijchp.2014.11.003] [Medline: 30487829]

188. de Oliveira-Filho AD, Morisky DE, Neves SJF, Costa FA, de Lyra DP. The 8-item Morisky medication adherence scale: validation of a Brazilian-Portuguese version in hypertensive adults. Res Social Adm Pharm 2014;10(3):554-561. [doi: 10.1016/j.sapharm.2013.10.006] [Medline: 24268603]

189. DiBonaventura M, Wintfeld N, Huang J, Goren A. The association between nonadherence and glycated hemoglobin among type 2 diabetes patients using basal insulin analogs. Patient Prefer Adherence 2014;8:873-882 [FREE Full text] [doi: 10.2147/PPA.S55550] [Medline: 24971002]

190. Fabbrini G, Abbruzzese G, Barone P, Antonini A, Tinazzi M, Castegnaro G, REASON study group. Adherence to anti-Parkinson drug therapy in the 'REASON' sample of Italian patients with Parkinson's disease: the linguistic validation of the Italian version of the 'Morisky medical adherence scale-8 items'. Neurol Sci 2013 Nov;34(11):2015-2022. [doi: 10.1007/s10072-013-1438-1] [Medline: 23728715]

191. George J, Vuong T, Bailey MJ, Kong DC, Marriott JL, Stewart K. Medication regimen complexity and adherence in patients at risk of medication misadventure. J Pharm Pract Res 2015 Apr 13;36(2):99-102. [doi: 10.1002/j.2055-2335.2006.tb00580.x]

192. Jankowska-Polanska B, Uchmanowicz I, Chudiak A, Dudek K, Morisky D, Szymanska-Chabowska A. Psychometric properties of the Polish version of the eight-item Morisky medication adherence scale in hypertensive adults. Patient Prefer Adherence 2016;10:1759-1766 [FREE Full text] [doi: 10.2147/PPA.S101904] [Medline: 27672314]

193. Kim J, Lee W, Hong Y, Ryu W, Lee KJ, Lee W, et al. Psychometric properties of a short self-reported measure of medication adherence among patients with hypertension treated in a busy clinical setting in Korea. J Epidemiol 2014;24(2):132-140 [FREE Full text] [doi: 10.2188/jea.je20130064] [Medline: 24463958]

194. Korb-Savoldelli V, Gillaizeau F, Pouchot J, Lenain E, Postel-Vinay N, Plouin PF, et al. Validation of a French version of the 8-item Morisky medication adherence scale in hypertensive adults. J Clin Hypertens (Greenwich) 2012 Jul;14(7):429-434 [FREE Full text] [doi: 10.1111/j.1751-7176.2012.00634.x] [Medline: 22747615]

195. Lee W, Ahn J, Kim J, Hong Y, Hong SK, Kim YT, et al. Reliability and validity of a self-reported measure of medication adherence in patients with type 2 diabetes mellitus in Korea. J Int Med Res 2013 Aug;41(4):1098-1110 [FREE Full text] [doi: 10.1177/0300060513484433] [Medline: 23860015] 
196. Morisky DE, Ang A, Krousel-Wood M, Ward HJ. Predictive validity of a medication adherence measure in an outpatient setting. J Clin Hypertens (Greenwich) 2008 May;10(5):348-354 [FREE Full text] [doi: 10.1111/j.1751-7176.2008.07572.x] [Medline: 18453793]

197. Nakhaeizadeh M, Khalooei A. Psychometric properties of Persian version of the 8-item Morisky medication adherence scale in type 2 diabetes patients. J Clin Diagn Res 2019. [doi: 10.7860/jcdr/2019/41408.13076]

198. Sakthong P, Chabunthom R, Charoenvisuthiwongs R. Psychometric properties of the Thai version of the 8-item Morisky medication adherence scale in patients with type 2 diabetes. Ann Pharmacother 2009 May;43(5):950-957. [doi: 10.1345/aph.1L453] [Medline: 19366872]

199. Shilbayeh SA, Almutairi WA, Alyahya SA, Alshammari NH, Shaheen E, Adam A. Validation of knowledge and adherence assessment tools among patients on warfarin therapy in a Saudi hospital anticoagulant clinic. Int J Clin Pharm 2018 Feb;40(1):56-66. [doi: 10.1007/s11096-017-0569-5] [Medline: 29189976]

200. Tan C, Teng G, Chong K, Cheung P, Lim A, Wee H, et al. Utility of the Morisky medication adherence scale in gout: a prospective study. Patient Prefer Adherence 2016;10:2449-2457 [FREE Full text] [doi: 10.2147/PPA.S119719] [Medline: 27980395]

201. Wang Y, Kong M, Ko Y. Psychometric properties of the 8-item Morisky medication adherence scale in patients taking warfarin. Thromb Haemost 2012 Oct;108(4):789-795. [doi: 10.1160/TH12-05-0368] [Medline: 22836920]

202. Zongo A, Guénette L, Moisan J, Guillaumie L, Lauzier S, Grégoire JP. Revisiting the internal consistency and factorial validity of the 8-item Morisky medication adherence scale. SAGE Open Med 2016;4:2050312116674850 [FREE Full text] [doi: 10.1177/2050312116674850] [Medline: 27895914]

203. Osborn CY, Gonzalez JS. Measuring insulin adherence among adults with type 2 diabetes. J Behav Med 2016 Aug 9;39(4):633-641 [FREE Full text] [doi: 10.1007/s10865-016-9741-y] [Medline: 27062271]

204. Arnet I, Metaxas C, Walter PN, Morisky DE, Hersberger KE. The 8-item Morisky medication adherence scale translated in German and validated against objective and subjective polypharmacy adherence measures in cardiovascular patients. $\mathrm{J}$ Eval Clin Pract 2015 Apr;21(2):271-277. [doi: 10.1111/jep.12303] [Medline: 25558796]

205. Shin D, Kim C. Psychometric evaluation of a Korean version of the 8-item medication adherence scale in rural older adults with hypertension. Aust J Rural Health 2013 Dec;21(6):336-342. [doi: 10.1111/ajr.12070] [Medline: 24299439]

206. Okello S, Nasasira B, Muiru AN, Muyingo A. Validity and reliability of a self-reported measure of antihypertensive medication adherence in Uganda. PLoS One 2016;11(7):e0158499 [FREE Full text] [doi: 10.1371/journal.pone.0158499] [Medline: 27367542]

207. Reynolds K, Viswanathan HN, Muntner P, Harrison TN, Cheetham TC, Hsu JY, et al. Validation of the osteoporosis-specific Morisky medication adherence scale in long-term users of bisphosphonates. Qual Life Res 2014 Sep;23(7):2109-2120. [doi: 10.1007/s11136-014-0662-3] [Medline: 24604077]

208. Reynolds K, Viswanathan HN, O'Malley CD, Muntner P, Harrison TN, Cheetham TC, et al. Psychometric properties of the osteoporosis-specific Morisky medication adherence scale in postmenopausal women with osteoporosis newly treated with bisphosphonates. Ann Pharmacother 2012 May;46(5):659-670. [doi: 10.1345/aph.1Q652] [Medline: 22510666]

209. Wickersham KE, Sereika SM, Kang H, Tamres LK, Erlen JA. Use of a self-report medication adherence scale for measuring adherence to antiretroviral therapy in patients with HIV/AIDS. J Nurs Meas 2018 Aug;26(2):E72-E88. [doi: 10.1891/1061-3749.26.2.E72] [Medline: $\underline{\text { 30567952] }}$

210. Athavale AS, Bentley JP, Banahan BF, McCaffrey DJ, Pace PF. Preliminary development of the medication nonpersistence scale. J Am Pharm Assoc (2003) 2017;57(3):389-94.e1. [doi: 10.1016/j.japh.2017.01.014] [Medline: 28279599]

211. Resnick B, Wehren L, Orwig D. Reliability and validity of the self-efficacy and outcome expectations for osteoporosis medication adherence scales. Orthop Nurs 2003;22(2):139-147. [doi: 10.1097/00006416-200303000-00012] [Medline: 12703398]

212. Qi B, Resnick B. Reliability and validity of the Chinese versions of self-efficacy and outcome expectations for osteoporosis medication adherence scales in Chinese immigrants. J Nurs Meas 2014;22(3):472-488. [doi: 10.1891/1061-3749.22.3.472] [Medline: 25608433]

213. Almeida-Brasil CC, Nascimento ED, Silveira MR, Bonolo PD, Ceccato MD. New patient-reported outcome measure to assess perceived barriers to antiretroviral therapy adherence: the PEDIA scale. Cad Saude Publica 2019;35(5):e00184218 [FREE Full text] [doi: 10.1590/0102-311x00184218] [Medline: $\underline{\text { 31166419] }}$

214. Kalichman SC, Cain D, Fuhrel A, Eaton L, di Fonzo K, Ertl T. Assessing medication adherence self-efficacy among low-literacy patients: development of a pictographic visual analogue scale. Health Educ Res 2005 Feb;20(1):24-35. [doi: 10.1093/her/cyg106] [Medline: 15253999]

215. Zschocke I, Mrowietz U, Lotzin A, Karakasili E, Reich K. Assessing adherence factors in patients under topical treatment: development of the topical therapy adherence questionnaire (TTAQ). Arch Dermatol Res 2014 Apr;306(3):287-297 [FREE Full text] [doi: 10.1007/s00403-014-1446-x] [Medline: 24509981]

216. Arnsten J, Demas P, Farzadegan H, Grant R, Gourevitch M, Chang C, et al. Antiretroviral therapy adherence and viral suppression in HIV-infected drug users: comparison of self-report and electronic monitoring. Clin Infect Dis 2001 Oct 15;33(8):1417-1423 [FREE Full text] [doi: 10.1086/323201] [Medline: 11550118] 
217. Teixeira A, Oliveira C, Teixeira M, Gaio AR, Lobo JM, de Almeida IF, et al. Development and validation of a novel questionnaire for adherence with topical treatments in psoriasis (QATOP). Am J Clin Dermatol 2017 Aug;18(4):571-581. [doi: 10.1007/s40257-017-0272-2] [Medline: 28321796]

218. Walewski KM, Cicutto L, D'Urzo AD, Heslegrave RJ, Chapman KR. Evaluation of a questionnaire to assess compliance with anti-asthma medications. J Asthma 2004 Feb;41(1):77-83. [doi: 10.1081/jas-120026064] [Medline: 15046381]

219. Lewin AB, LaGreca AM, Geffken GR, Williams LB, Duke DC, Storch EA, et al. Validity and reliability of an adolescent and parent rating scale of type 1 diabetes adherence behaviors: the self-care inventory (SCI). J Pediatr Psychol 2009 Oct;34(9):999-1007 [FREE Full text] [doi: 10.1093/jpepsy/jsp032] [Medline: 19423660]

220. Mumtaz T, Haider SA, Malik JA, La Greca AM. Translation, validation and effectiveness of self-care inventory in assessing adherence to diabetes treatment. J Pak Med Assoc 2016 Jul;66(7):853-858 [FREE Full text] [Medline: 27427135]

221. Jansà M, Vidal, Gimenez, Conget, Gallindo M, Roca, Colungo, Esmatjes, Salamero. Psychometric analysis of the Spanish and Catalan versions of the Diabetes Self-Care Inventory-Revised version questionnaire. PPA 2013 Oct:997. [doi: 10.2147/ppa.s50271]

222. Alhomoud F, Alhomoud F, Millar I. How effectively are your patients taking their medicines? A critical review of the strathclyde compliance risk assessment tool in relation to the 'MMAS' and 'MARS'. J Eval Clin Pract 2016 Jun;22(3):411-420. [doi: 10.1111/jep.12501] [Medline: 26696012]

223. Lubinga SJ, Millar I, Babigumira JB. Pilot evaluation of the psychometric properties of a self-medication risk assessment tool among elderly patients in a community setting. BMC Res Notes 2011 Oct 11;4:398 [FREE Full text] [doi: 10.1186/1756-0500-4-398] [Medline: 21989334]

224. Pedrosa RB, Rodrigues RC, Oliveira HC, Alexandre NM. Construct validity of the Brazilian version of the self-efficacy for appropriate medication adherence scale. J Nurs Meas 2016;24(1):E18-E31. [doi: 10.1891/1061-3749.24.1.E18] [Medline: 27103240]

225. Pedrosa RB, Rodrigues RC. Adaptation and evaluation of the measurement properties of the Brazilian version of the self-efficacy for appropriate medication adherence scale. Rev Lat Am Enfermagem 2016;24:e2692 [FREE Full text] [doi: 10.1590/1518-8345.0167.2692] [Medline: 27192417]

226. Risser J, Jacobson TA, Kripalani S. Development and psychometric evaluation of the self-efficacy for appropriate medication use scale (SEAMS) in low-literacy patients with chronic disease. J Nurs Meas 2007;15(3):203-219. [doi: 10.1891/106137407783095757] [Medline: 18232619]

227. Najimi A, Mostafavi F, Sharifirad G, Golshiri P. Development and study of self-efficacy scale in medication adherence among Iranian patients with hypertension. J Educ Health Promot 2017;6:83 [FREE Full text] [doi: 10.4103/jehp.jehp 64 16] [Medline: 29114551]

228. Blumberg EJ, Hovell MF, Kelley NJ, Vera AY, Sipan CL, Berg JP. Self-report INH adherence measures were reliable and valid in Latino adolescents with latent tuberculosis infection. J Clin Epidemiol 2005 Jun;58(6):645-648. [doi: 10.1016/j.jclinepi.2004.11.022] [Medline: 15878479]

229. Muñoz-Moreno JA, Fumaz CR, Ferrer MJ, Tuldrà A, Rovira T, Viladrich C, SERAD Validation Team. Assessing self-reported adherence to HIV therapy by questionnaire: the SERAD (self-reported adherence) study. AIDS Res Hum Retroviruses 2007 Oct;23(10):1166-1175. [doi: 10.1089/aid.2006.0120] [Medline: 17961100]

230. Rofail D, Abetz L, Viala M, Gait C, Baladi J, Payne K. Satisfaction and adherence in patients with iron overload receiving iron chelation therapy as assessed by a newly developed patient instrument. Value Health 2009;12(1):109-117 [FREE Full text] [doi: 10.1111/j.1524-4733.2008.00390.x] [Medline: 18637142]

231. Knobel H, Alonso J, Casado JL, Collazos J, González J, Ruiz I, GEEMA Study Group. Validation of a simplified medication adherence questionnaire in a large cohort of HIV-infected patients: the GEEMA study. AIDS 2002 Mar 8;16(4):605-613. [doi: 10.1097/00002030-200203080-00012] [Medline: 11873004]

232. Ortega Suárez FJ, Sánchez Plumed J, Pérez Valentín MA, Pereira Palomo P, Muñoz Cepeda MA, Lorenzo Aguiar D, Grupo de Estudio Vatren. Validation on the simplified medication adherence questionnaire (SMAQ) in renal transplant patients on tacrolimus. Nefrologia 2011;31(6):690-696 [FREE Full text] [doi: 10.3265/Nefrologia.pre2011.Aug.10973] [Medline: 22130285]

233. Rathbun RC, Farmer KC, Lockhart SM, Stephens JR. Validity of a stage of change instrument in assessing medication adherence in indigent patients with HIV infection. Ann Pharmacother 2007 Feb;41(2):208-214. [doi: 10.1345/aph.1H383] [Medline: 17213294$]$

234. Willey C, Redding C, Stafford J, Garfield F, Geletko S, Flanigan T, et al. Stages of change for adherence with medication regimens for chronic disease: development and validation of a measure. Clin Ther 2000 Jul;22(7):858-871. [doi: 10.1016/s0149-2918(00)80058-2] [Medline: 10945512]

235. Julian FS, Martin P, Erickson SR. Validation of the special projects of national significance adherence tool in HIV/AIDS patients. Ann Pharmacother 2010 May 4;44(6):1003-1009. [doi: 10.1345/aph.1m690]

236. Lu M, Safren SA, Skolnik PR, Rogers WH, Coady W, Hardy H, et al. Optimal recall period and response task for self-reported HIV medication adherence. AIDS Behav 2008 Jan;12(1):86-94. [doi: 10.1007/s10461-007-9261-4] [Medline: 17577653]

237. Gutiérrez-Pereyra F, Plaza V, Fernández-Rodríguez C. Validation of the 'test of the adherence to inhalers' (TAI) for asthma and COPD patients. - 2020:-. [doi: 10.1183/13993003.congress-2015.pa5012] 
238. Mancebo MC, Pinto A, Rasmussen SA, Eisen JL. Development of the treatment adherence survey-patient version (TAS-P) for OCD. J Anxiety Disord 2008;22(1):32-43 [FREE Full text] [doi: 10.1016/j.janxdis.2007.01.009] [Medline: 17324553]

239. He W, Bonner A, Anderson D. Patient reported adherence to hypertension treatment: a revalidation study. Eur J Cardiovasc Nurs 2016 Apr;15(2):150-156. [doi: 10.1177/1474515115603902] [Medline: 26307159]

240. Giordano TP, Guzman D, Clark R, Charlebois ED, Bangsberg DR. Measuring adherence to antiretroviral therapy in a diverse population using a visual analogue scale. HIV Clin Trials 2004;5(2):74-79. [doi: 10.1310/JFXH-G3X2-EYM6-D6UG] [Medline: 15116282]

241. Duncan N, Kronenberger W, Roberson C, Shapiro A. VERITAS-PRN: a new measure of adherence to episodic treatment regimens in haemophilia. Haemophilia 2010 Jan;16(1):47-53. [doi: 10.1111/j.1365-2516.2009.02094.x] [Medline: 19754842]

242. Duncan N, Kronenberger W, Roberson C, Shapiro A. VERITAS-Pro: a new measure of adherence to prophylactic regimens in haemophilia. Haemophilia 2010 Mar;16(2):247-255. [doi: 10.1111/j.1365-2516.2009.02129.x] [Medline: 19925631]

243. Liau YW, Cheow C, Leung KTY, Tan H, Low SF, Cheen HH, et al. A cultural adaptation and validation study of a self-report measure of the extent of and reasons for medication nonadherence among patients with diabetes in Singapore. Patient Preference Adherence 2019 Jul;Volume 13:1241-1252. [doi: 10.2147/ppa.s208736]

244. Blalock DV, Zullig LL, Bosworth HB, Taylor SS, Voils CI. Self-reported medication nonadherence predicts cholesterol levels over time. J Psychosom Res 2019 Mar;118:49-55. [doi: 10.1016/j.jpsychores.2019.01.010] [Medline: 30782354]

245. Vreeman RC, Scanlon ML, Tu W, Slaven JE, McAteer CI, Kerr SJ, et al. Validation of a self-report adherence measurement tool among a multinational cohort of children living with HIV in Kenya, South Africa and Thailand. J Int AIDS Soc 2019 May;22(5):e25304 [FREE Full text] [doi: 10.1002/jia2.25304] [Medline: 31148372]

246. Vale FC, Santa-Helena ET, Santos MA, Carvalho WM, Menezes PR, Basso CR, et al. Development and validation of the webAd-Q questionnaire to monitor adherence to HIV therapy. Rev Saude Publica 2018;52:62 [FREE Full text] [doi: 10.11606/s1518-8787.2018052000337] [Medline: 29846437]

247. Phillips T, Brittain K, Mellins CA, Zerbe A, Remien RH, Abrams EJ, et al. A self-reported adherence measure to screen for elevated HIV viral load in pregnant and postpartum women on antiretroviral therapy. AIDS Behav 2017 Feb;21(2):450-461 [FREE Full text] [doi: 10.1007/s10461-016-1448-0] [Medline: 27278548]

248. Wilson IB, Lee Y, Michaud J, Fowler FJ, Rogers WH. Validation of a new three-item self-report measure for medication adherence. AIDS Behav 2016 Nov;20(11):2700-2708 [FREE Full text] [doi: 10.1007/s10461-016-1406-x] [Medline: 27098408]

249. Fredericksen R, Yang F, Gibbons L, Edwards T, Brown S, Fitzsimmons E, et al. Development and content validation of measures assessing adherence barriers and behaviors for use in clinical care. Res Social Adm Pharm 2019 Sep;15(9):1168-1176. [doi: 10.1016/j.sapharm.2018.10.001] [Medline: 30327183]

250. Linn AJ, Vervloet M, van Dijk L, Smit EG, van Weert JC. Effects of eHealth interventions on medication adherence: a systematic review of the literature. J Med Internet Res 2011 Dec 5;13(4):e103 [FREE Full text] [doi: 10.2196/jmir.1738] [Medline: 22138112]

251. Muntner P, Joyce C, Holt E, He J, Morisky D, Webber LS, et al. Defining the minimal detectable change in scores on the eight-item Morisky medication adherence scale. Ann Pharmacother 2011 May;45(5):569-575. [doi: 10.1345/aph.1P677] [Medline: 21521862]

252. Stoll CR, Izadi S, Fowler S, Green P, Suls J, Colditz GA. The value of a second reviewer for study selection in systematic reviews. Res Synth Methods 2019 Dec;10(4):539-545. [doi: 10.1002/jrsm.1369] [Medline: 31272125]

\section{Abbreviations}

AACTG: Adult AIDS Clinical Trials Group

ASK-20: Adherence Starts with Knowledge 20

COSMIN: Consensus-based Standards for the selection of health Measurement INstruments

CQR: Compliance Questionnaire-Rheumatology

GMAS: General Medication Adherence Scale

ITBS: Immunosuppressant Therapy Barrier Scale

MAR-Scale: Medication Adherence Reasons Scale

MARS: Medication Adherence Rating Scale

MARS-5: five-item Medication Adherence Rating Scale

MARS-9: nine-item Medication Adherence Rating Scale

MMAS-4: four-item Morisky Medication Adherence Scale

MMAS-8: eight-item Morisky Medication Adherence Scale

PROM: patient-reported outcome measure

PULSES: Population-based, Unified, Learning System for Enhances and Sustainable Health

SEAMS: self-efficacy for appropriate medication adherence scale

SICT: satisfaction with iron chelation therapy

TAI: Test of Adherence to Inhalers 


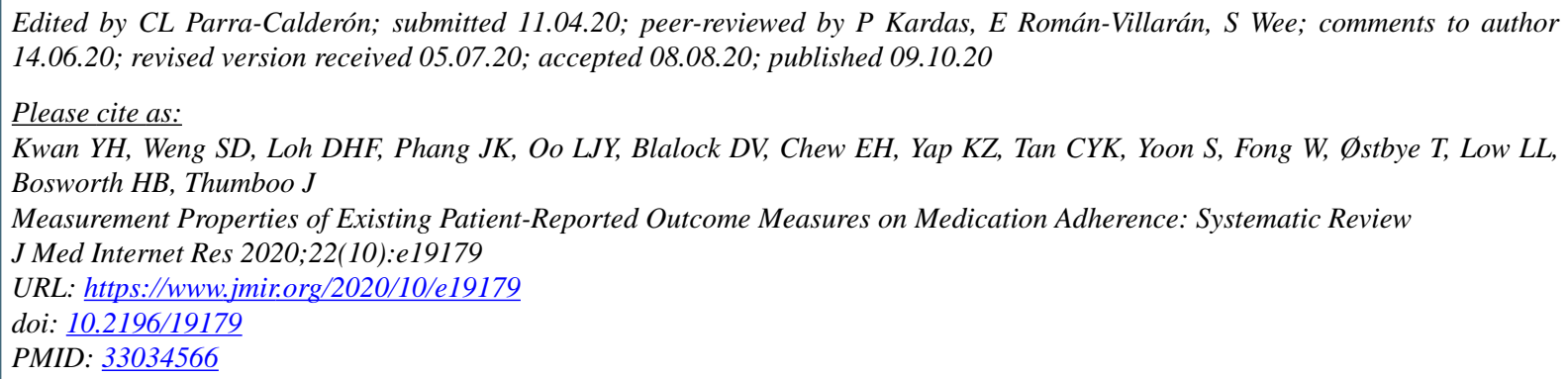

(CYu Heng Kwan, Si Dun Weng, Dionne Hui Fang Loh, Jie Kie Phang, Livia Jia Yi Oo, Dan V Blalock, Eng Hui Chew, Kai Zhen Yap, Corrinne Yong Koon Tan, Sungwon Yoon, Warren Fong, Truls Østbye, Lian Leng Low, Hayden Barry Bosworth, Julian Thumboo. Originally published in the Journal of Medical Internet Research (http://www.jmir.org), 09.10.2020. This is an open-access article distributed under the terms of the Creative Commons Attribution License (https://creativecommons.org/licenses/by/4.0/), which permits unrestricted use, distribution, and reproduction in any medium, provided the original work, first published in the Journal of Medical Internet Research, is properly cited. The complete bibliographic information, a link to the original publication on http://www.jmir.org/, as well as this copyright and license information must be included. 